\title{
Piezo Scanning
}

\section{James Litynski and Andreas Blume}

Piezosystem Jena, Inc.

Hopedale, Massachusetts, USA

\section{CONTENTS}

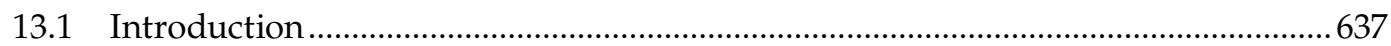

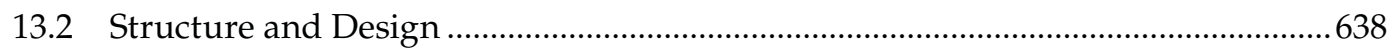

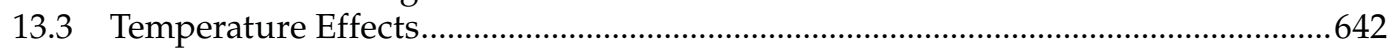

13.4 Properties of Motion...........................................................................................643

13.5 Properties of Stack-Flexure Structures ……………………………………….....645

13.6 Electrical Drives .............................................................................................648

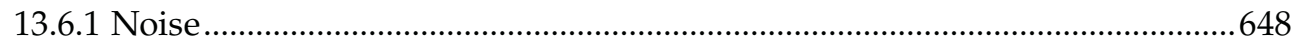

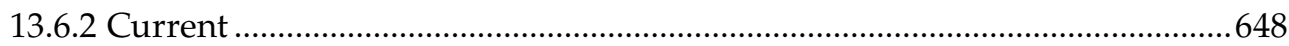

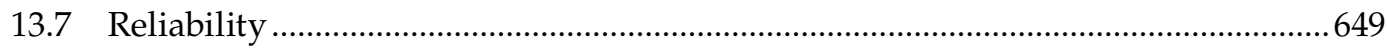

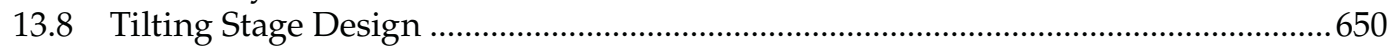

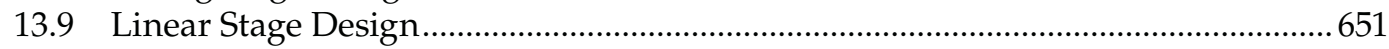

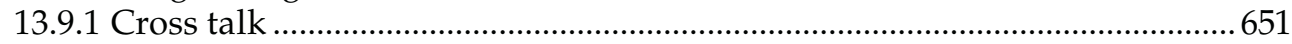

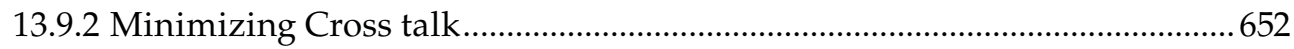

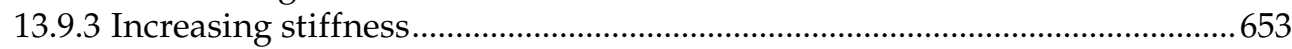

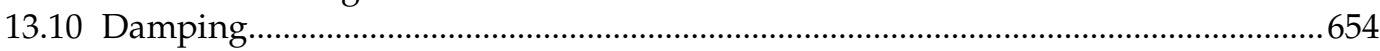

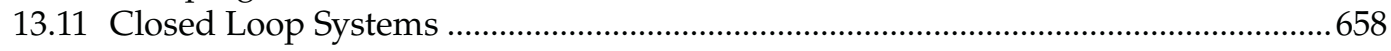

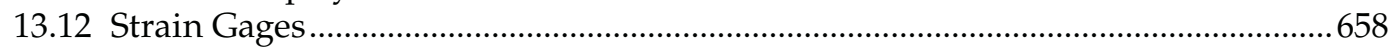

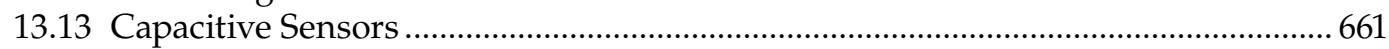

13.14 Electronic Control Architecture For Closed Loop Systems.........................................662

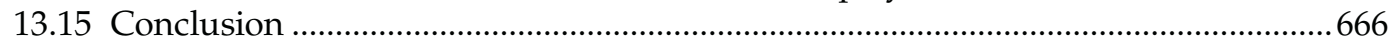

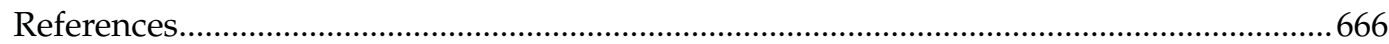

\subsection{INTRODUCTION}

The piezoelectric effect is the production of positive and negative charges on the surface of certain types of crystalline structures in the presence of an externally applied force that changes the shape of the crystal. The converse is also true. When positive and negative charges are applied to the surface of these same crystals, forces are produced within the crystals which change its shape. This inverse piezoelectric effect has recently been used in micropositioning devices to good effect. In low voltage $(<200 \mathrm{~V})$ applications, a potential of $-20 \mathrm{~V}$ to $150 \mathrm{~V}$ is applied to bonded stacks of such crystals so that they expand. With current piezoelectric stacks available commercially today, such expansion is on the order of $1 \mu \mathrm{m}$ for every $1 \mathrm{~mm}$ of stacked piezoelectric crystal. Due to this rather small expansion, and the unpredictable trajectory of the crystal faces, flexure hinges are commonly used to 
both amplify and guide the motion in a piezo stage. Major advances in stage design within the past 15 years have resulted in a large variety of scanning and fine positioning devices which take advantage of the rather unique properties of this type of drive mechanism.

Piezoelectric flexure drive mechanisms offer a number of advantages over traditional stepping and servo motor bearing guide stages. One of the main advantages of this type of drive is their very high resolution. Since the entire flexure mechanism is driven by the smooth expansion of a crystal, it is a friction and stiction free system with a resolution that is only limited by the electrical noise on the voltage potential being applied to create the inverse piezoelectric effect and the mechanical noise that may affect the flexure structure from the environment. This has been the driving factor behind the development of extremely lownoise amplifiers and highly stiff, robust mechanical flexures with high resonant frequencies to keep outside vibrations that may excite the stage to a minimum. A second advantage of piezoelectric stages is the crystal's ability to exert extremely high forces. A $25-\mathrm{mm}^{2}$ area of PZT (lead zirconium titanate) can exert a force in excess of $1000 \mathrm{~N}$. A third advantage of piezoelectric stages is their high resonant frequency. PZT preloaded stacks by themselves may safely operate up to $75 \mathrm{kHz}$ and, when integrated into a flexure mechanism, $4 \mathrm{kHz}$ is not uncommon for a tilting stage, making them very useful in laser scanning applications.

A number of obstacles present themselves in practice when using piezoelectric materials in a laser scanning system. These include a large hysteresis in voltage versus position, drift or creep, unpredictable force vectors during crystal expansion, temperature limitations due to the Curie temperature and reduction of the piezoelectric effect at low temperatures, environmental noise amplification by the flexure design, low tolerance for tensile forces, and electrical failure due to ion migration at the electrical contacts.

The object of this chapter will be to give a practical understanding of the use of these devices allowing the scientist or engineer to make the most of their inherent advantages while addressing the constraints they impose.

\subsection{STRUCTURE AND DESIGN}

Piezoelectric ceramics exhibit a Perovskite ionic lattice structure $\left(\mathrm{AXO}_{3}\right)$. Below their Curie temperature these types of crystals exhibit an inherent polarization. This is caused by a deviation of the titanium ion from its center position within the crystal lattice which creates an electrical dipole.

Under normal conditions such a ceramic exhibits an anisotropic structure and all the dipoles within the material domains of the ceramic are oriented randomly. However, in the presence of a strong electric field these domains become ferroelectric. The dipoles all align in the direction of the electric field. This effect is long term. After removal of this strong electric field, the structure relaxes but remains strongly polarized (Figure 13.1).

Depending on the orientation of the electric field to the crystal structure, forces generation by the piezoceramic can be characterized as longitudinal $d_{33}$, transverse $d_{31}$ or shear $\mathrm{d}_{15}$. The transverse mode is used in bi-morph (bending) and tube piezoelectric actuators. Stacked-type actuators use the longitudinal mode for expansion. To somewhat limit the scope of this chapter, we will concentrate on theory and devices which take advantage of the longitudinal mode.

Modern piezo stack structures consist of hundreds of thin layers of PZT with a thickness of approximately $100 \mu \mathrm{m}$. Electrodes are arranged on opposite sides of the stack structure 


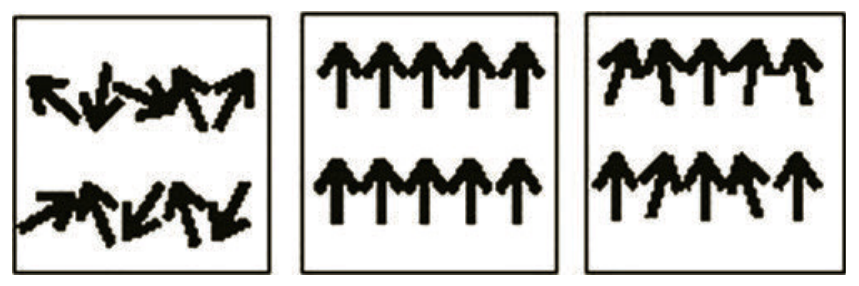

FIGURE 13.1

Domain polarization randomly ordered, saturated, and remanent (from left to right).

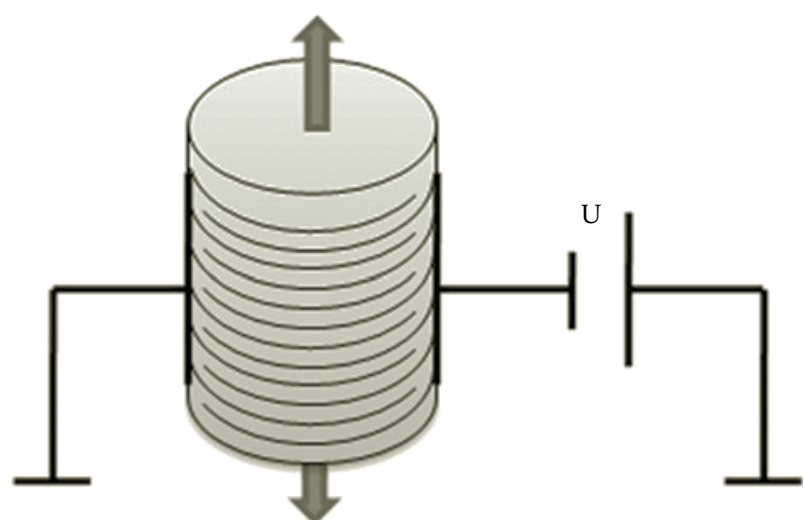

FIGURE 13.2

Construction of a piezo stack.

with connections to deposited metallic film such that alternating charged and grounded plates are sandwiched between the crystal stacks (Figure 13.2). The very thin layers of PZT require relatively low voltages in order for all dipoles to become completely aligned. A reversal in the polarity of these plates and therefore the direction of the electric field can result in some contraction of the crystalline structure, but the majority of the motion can be achieved by expansion.

Imagine the domain dipoles as arrows which orientate along the direction of the electric field. Applying a high field means the arrow stands vertically, head on top (maximum stroke). Without the electrical field the arrow lays horizontally (minimum stroke). A reversal of the polarity of the electric field lets the arrow stand also, but head on bottom (maximum stroke) (Figure 13.3).

This is caused by the essential deviation of the titanium ion. In reality the "arrows" rotate only a few degrees. Saturation may exist even when all "arrows" are not aligned exactly parallel to each other; most of the "arrows " reached the maximum position early and some later, but the magnitude of the whole stroke remains the same for a given time period.

Many devices take advantage of the extended range offered by this contraction and hence most power supplies offer a voltage range which extends down to -20 V. Stack manufacturers recommend upper voltage limits of $100 \mathrm{~V}$ to $150 \mathrm{~V}$. This limit is imposed by the breakthrough field strength of the ceramic and is affected by the thicknesses of the layers. 


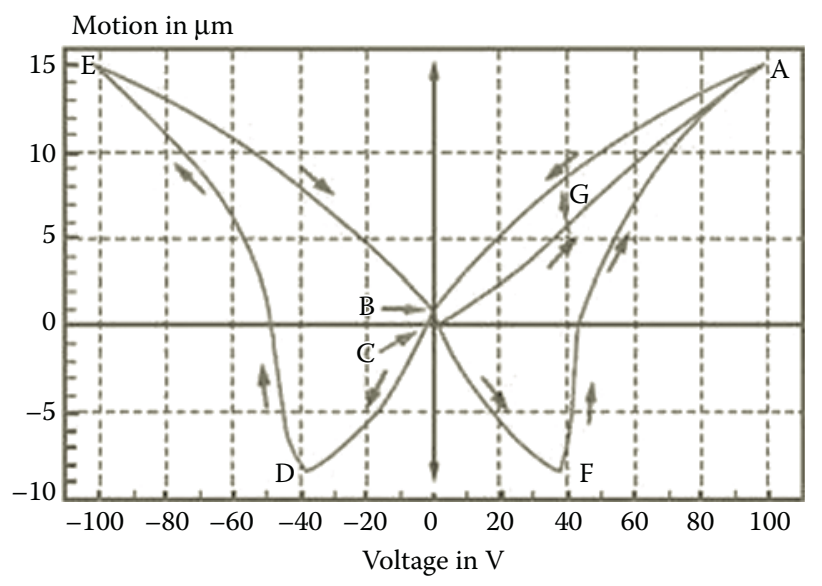

FIGURE 13.3

According to the applied voltage, the motion of a piezo element will follow the points ABCDEF.

The physical structure of these stacks-layers of charged metal plates sandwiched between an insulating material is, of course, the classical structure of a parallel-plate capacitor and, as you might imagine, these stacks have a quite large capacitance.

Solving for the capacitance of a parallel-plate capacitor we have the formula:

$$
C=\left(\varepsilon_{\mathrm{o}} \varepsilon_{r} A\right) / d
$$

where $A$ is the area of the plate, and $d$ is the distance between them. The material specific relative permittivity $\varepsilon_{r}$ takes into account the charge bonded by the ceramic material (dielectric) and greatly increases the capacitance.

From this formula the capacitance of a single layer of $5 \mathrm{~mm} \times 5 \mathrm{~mm}$ PZT can be calculated:

$$
C=(8.9 \mathrm{E}-12) F / m^{*} 50 E 6^{*}(25 \mathrm{E}-6) m^{2} / 100(\mathrm{E}-6) m
$$

And therefore a 10-mm stack of 100 such disks would have a capacitance of appr. $1.0 \mu \mathrm{F}$ at a $50-\mathrm{Hz}$ measuring frequency.

One might think from this calculation that $d$ is, in this case, a variable which depends on electric field strength which is directly proportional to the voltage applied to the metal plates within the stack. As the stack expands or contracts, this value will change accordingly. However, the change in $d$ for an application of $130 \mathrm{~V}$ is typically $0.1 \%(0.1 \mu \mathrm{m}$ for a $100-\mu \mathrm{m}$ layer) and for calculations concerning electrical requirements, this value is too small to be significant and other factors such as temperature and strain dependence are much larger than this. It should also be noted that current production methods for PZT layers are such that the thickness can vary quite a bit. Tolerance on stack thickness (and therefore capacitance, and motion/V (sensitivity)) can be as high as 10\%.

There are two different methods to prepare multilayer stacks. In the first method, discs are cut from a ceramic bulk, and then sandwiched between electrical contact films and epoxy glue. This is finished by a pressing process. In the second method the ceramic is powdered and applied to the contact film, layer by layer followed by sintering process (without epoxy). The tensile strength of the electrical contact/PZT bonds determines the tensile strength of the stack. 
This tensile strength is relatively weak compared to the compressive load that the stacks can endure. Under high dynamical operation internal accelerations inside the stack can generate forces which exceed the tensile strength limit of the bonds resulting in a delaminating of the layers. To prevent this, it is necessary to apply a preload to the stack of sufficient strength to overcome any internal force. A typical preload for a stack is $150 \mathrm{~N}$. Even with this strong preload it is possible to damage the stack if it, or the mechanical structure of which it is a part, is driven at resonant frequency with a large amplitude. It is therefore important to determine the resonant frequency first and try to operate below it if possible. Operating above the resonant frequency is possible for low amplitudes (I would recommend $<1 \%$ of the total motion), but of course the primary resonance will be excited in this case.

Since piezoelectric stacks are rather stiff and brittle it is important to take care when applying heavy loads or generating shock forces. The main concern here is to be sure that any load or force is applied directly on the same axis as the center of the stack. Off axis, shear loads or shock forces will typically crack the stack. A common strategy to minimize off-axis loading is to utilize a ball tip on the end of the stack or integrate the stack into a preloaded flexure which directs any outside force axially (Figures 13.4 and 13.5).
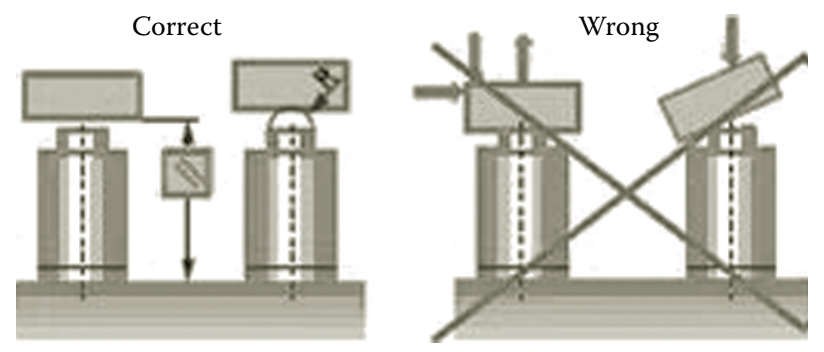

FIGURE 13.4

Applying load to a stack.

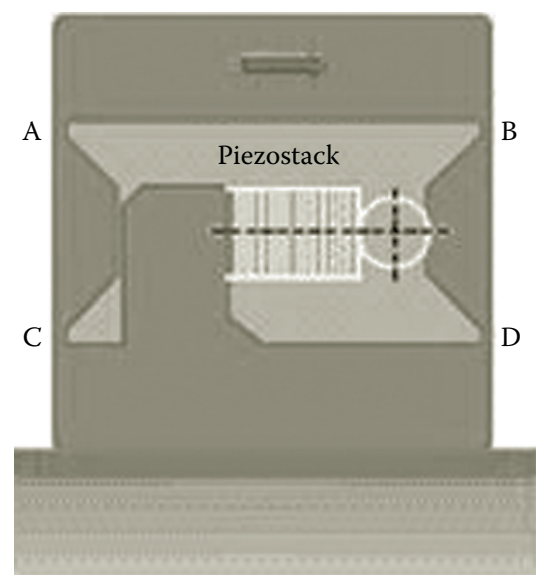

FIGURE 13.5

Integrated stack with flex hinges at ABCD. Lower bar is fixed and arrow indicates direction of motion. 


\subsection{TEMPERATURE EFFECTS}

Temperature variations play an extremely important role in micrometer scale positioning systems. Often a neophyte piezosystem user will complain that his system is "drifting all over the place" when, in fact, he is trying to measure on a scale where material expansions, invisible to the naked eye, are causing relatively huge shifts in position and he is simply witnessing his thermostat kicking the heat or air conditioning on and off.

Consider: The thermal coefficient of expansion for steel from $20^{\circ} \mathrm{C}-100^{\circ} \mathrm{C}$ is about $16 \mu \mathrm{m} / \mathrm{m}{ }^{\circ} \mathrm{C}$. A simple piezo flexure stage may incorporate up to $50 \mathrm{~mm}$ of steel in its design with specified resolutions for closed loop systems of $10 \mathrm{~nm}$ or less. A variation of just half a degree will result in an expansion of the steel of $400 \mathrm{~nm}$ - or more than $400 \times$ the resolution of the stage!

To demonstrate this effect I measured the position of a parallelogram flexure design with an integrated PZT stack under static voltage conditions over an extended period of time with a michaelson-type interferometer. Temperature measurements of the air were taken simultaneously and the results are shown in Figure 13.6.

An interesting feature of PZT is that it has a negative coefficient of expansion at room temperature of $-6 \mu \mathrm{m} / \mathrm{m}{ }^{\circ} \mathrm{C}$. The total displacement caused by thermal effects can be expressed by the following formula:

$$
d l_{\text {therm }} / d T=L_{\text {piezo }} * \alpha_{\text {piezo }}+L_{\text {metal }} * \alpha_{\text {metal }}
$$

To see the effect of this on a stack type actuator we can use the example of a 50 - $\mu \mathrm{m}$ stack which uses a combination of three 16-mm stacks in series (Table 13.1).

It is also possible to construct a simple temperature compensated block by selecting the proportionally correct amount of steel block and PZT and arranging them in series. So a stack that has a motion of $16 \mu \mathrm{m}$ (16 $\mathrm{mm}$ in length) would require a steel block of $6 \mathrm{~mm}$ to make a temperature compensated simple actuator. Materials with a higher temperature extension coefficient will decrease the additional length, for example, brass.

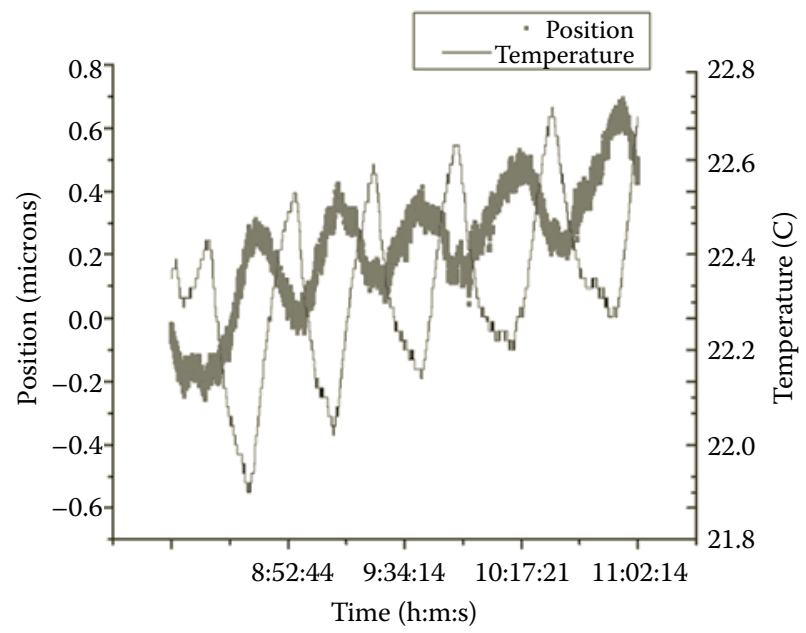

FIGURE 13.6

Temperature-induced motion on a $400-\mu \mathrm{m}$ flexure stage. 
TABLE 13.1

Thermal Effects on a PA 50 Preloaded Stack Actuator

All dimensions: $\mu \mathrm{m} /{ }^{\circ} \mathrm{C}$

\begin{tabular}{lccccccc}
\hline Temp & Displacement & $\begin{array}{c}\text { Thermal } \\
\text { expansion }^{\mathbf{a}}\end{array}$ & Sum & $\begin{array}{c}\text { Displ. three } \\
\text { stacks }\end{array}$ & $\begin{array}{c}\text { Therm exp. } \\
\text { three stacks }\end{array}$ & $\begin{array}{c}\text { Thermal } \\
\text { exp. steel }\end{array}$ & $\begin{array}{c}\text { Sum } \\
\text { actuator }\end{array}$ \\
\hline 30 & 16.1 & 0.0 & 16.1 & 48.3 & 0.0 & 0.0 & 48.3 \\
40 & 15.7 & -1.8 & 13.9 & 47.1 & -5.4 & 0.3 & 42.0 \\
60 & 15.3 & -3.5 & 11.8 & 45.9 & -10.5 & 0.9 & 36.3 \\
80 & 15.2 & -5.5 & 9.7 & 45.6 & -16.5 & 1.4 & 30.5 \\
100 & 14.6 & -7.4 & 7.2 & 43.8 & -22.2 & 2.0 & 23.6 \\
120 & 14.0 & -9.4 & 4.6 & 42.0 & -28.2 & 2.6 & 16.4 \\
\hline
\end{tabular}

a Thermal expansion of steel parts is calculated with $16^{*} 10^{-6 *} \mathrm{~K}^{-1}$ which causes an expansion of $30.4 \mathrm{~nm} / \mathrm{K}$.

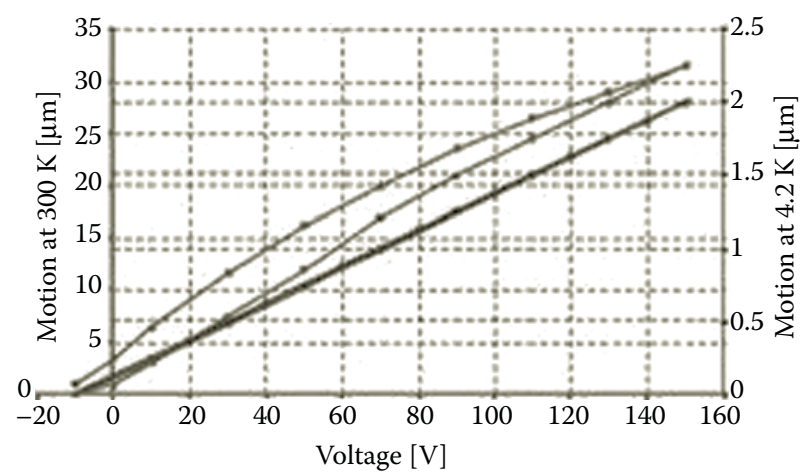

FIGURE 13.7

Piezo motion at room temperature (upper curve) and $4 \mathrm{~K}$ (lower curve).

Modern flexure designs utilize a variety of materials and methods for temperature compensation. But not all systems take temperature into consideration.

For most applications $\left(-10^{\circ} \mathrm{C}\right.$ to $\left.90^{\circ} \mathrm{C}\right)$ the piezoeffect is relatively constant. However, outside this temperature range the effect begins to decrease and at extremely low temperatures (i.e., $4 \mathrm{~K}$ ) the motion generated by an electric field on a stack can be as little as $6 \%$ of the motion generated at room temperature. The hysteresis curve of a stack which makes $32 \mu \mathrm{m}$ of motion is shown in Figure 13.7 for both room temperature and liquid helium operation.

Of course even this reduced motion can be very useful for many cryogenic studies and as you can see, the problem of hysteresis is reduced to the point where a closed loop system is often unnecessary.

\subsection{PROPERTIES OF MOTION}

Due to the discrete structure of a piezoelectric stack, its expansion is often a bit unpredictable. Stacks have a tendency to exhibit all kinds of bad behavior such as twisting and tilting. Additionally, the expansion is nonlinear and exhibits a great deal of hysteresis. 
Taken as a percentage of the full motion of the stack, this hysteresis can be as much as $12 \%$. To further complicate matters, this hysteresis is temperature and load dependent. At higher temperatures and under higher loads, this hysteresis will be even greater. Figure 13.8 shows the typical response of a piezoelectric to a triangle wave function.

Piezoelectric stacks also exhibit some drifting in position or "creep." This is an asymptotic decay toward a final position when given a step function which is a result of some crystal domains within the layers coming into alignment with the electric field more slowly. This creep is dependent on the expansion of the PZT, the external load, and time. It can be calculated as a logarithmic function with the following formula:

$$
d L / d t=d L_{0.1}[L+\gamma \lg (t / 0.1 \mathrm{~s})]
$$

$$
\begin{aligned}
& L=\text { length } \\
& t=\text { time } \\
& \begin{aligned}
d L_{0.1} & =\text { change in length after } 0.1 \mathrm{~s} \text { after the step function } \\
\gamma & =\text { drift constant (varies depending on loading, but typically 0.015) }
\end{aligned}
\end{aligned}
$$

A typical graph of creep over a period of $10 \mathrm{~min}$ is shown in Figure 13.9.

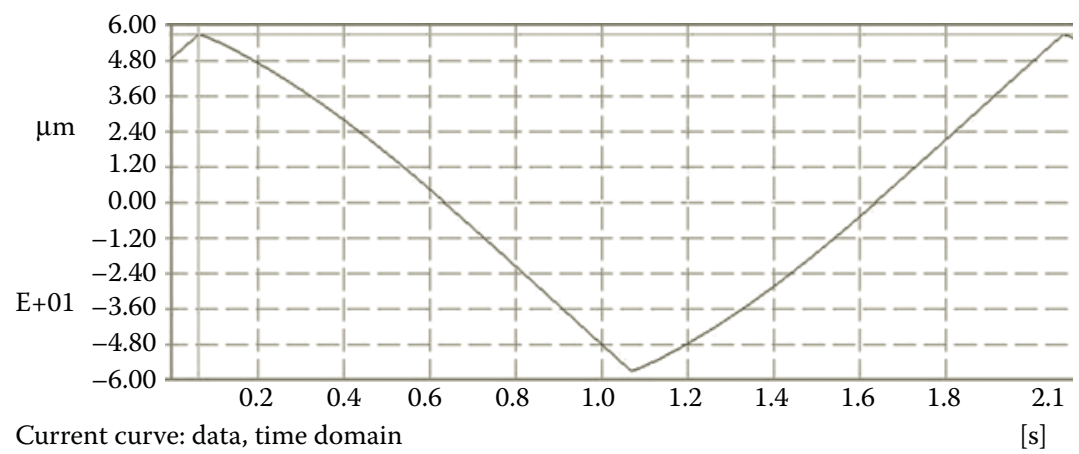

\section{FIGURE 13.8}

Piezoelectric stack total motion as a function of time as measured with an interferometer when driven with a triangle function at $0.5 \mathrm{~Hz}$.

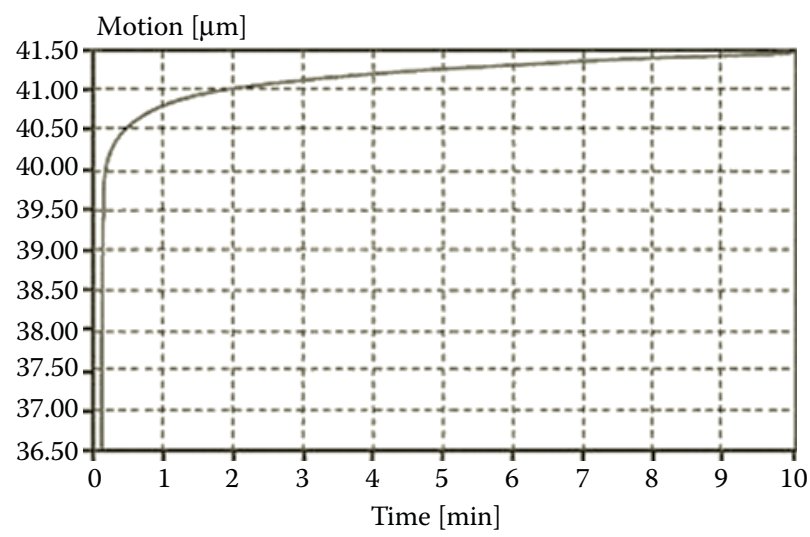

FIGURE 13.9

Creep exhibited by a PZT stack structure after given an electrical step function. 


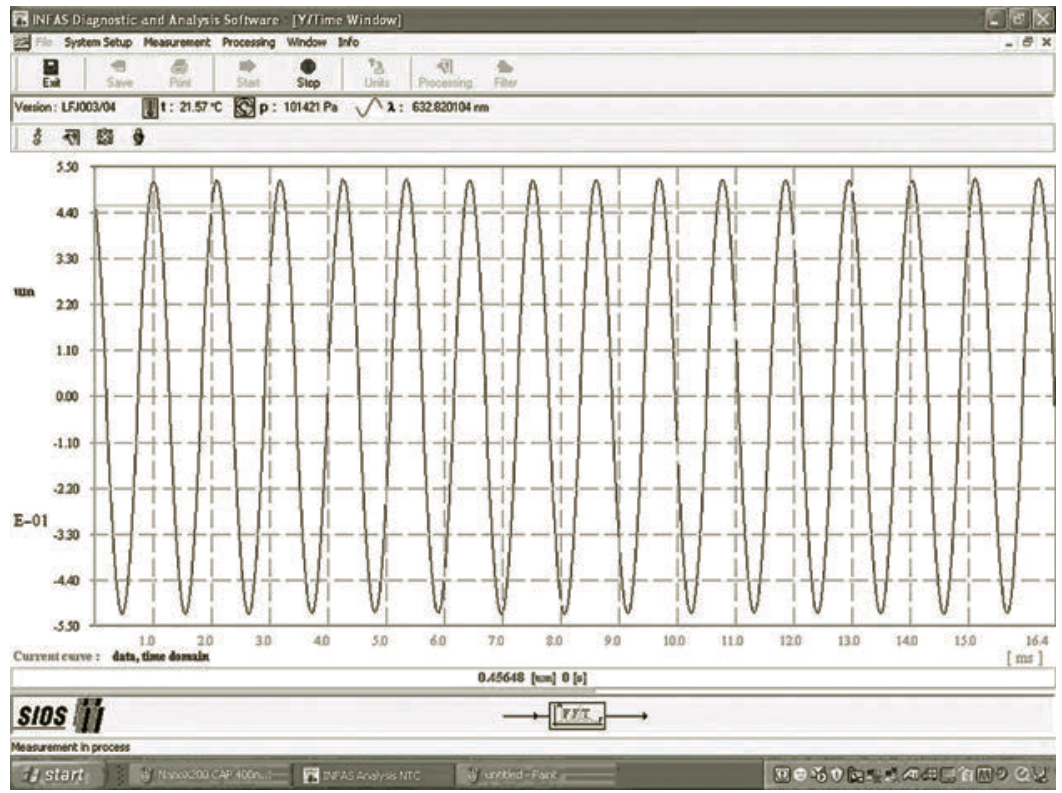

FIGURE 13.10

Demonstration of open loop repeatability of a PZT flexure.

A simple strategy often used for minimizing this creep is to overshoot the desired position by $5 \%$ or so and come back to it. This strategy brings the dipoles within the crystal structure more rapidly to their final rest state.

As you can see, the creep effect in piezosystems is long term and often negligible for dynamic operation. In fact, for high speed periodic signals piezosystems are highly repeatable. Although the function will still be subject to hysteresis, motion will follow the same track down to the noise of the system-often sub-nm. To provide an example, I drove a flexure stage capable of $240 \mu \mathrm{m}$ at a frequency of approximately $1 \mathrm{kHz}$ over a distance of $1 \mu \mathrm{m}$ with a sine wave function provided by a Tektronix function generator and amplified with a Piezosystem Jena power supply. Results were measured and graphed with a SIOS interferometric vibrometer system (Figure 13.10).

\subsection{PROPERTIES OF STACK-FLEXURE STRUCTURES}

The integration of piezoelectric stacks into solid state flexures has two major benefits when predicting behavior:

1. The structure can be easily described with spring formulae of classical dynamics.

2. Finite Element Analysis computer modeling can predict behavior under a variety of loading conditions.

For instance, we can estimate a change in natural resonant frequency when a stack-flexure structure is affected by an external load. Let's take the example of a stack-flexure stage from Piezosystem Jena shown in Figure 13.11. 


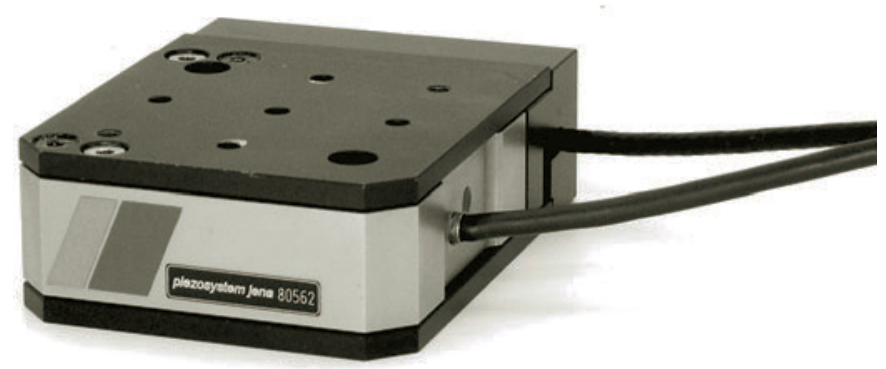

\section{FIGURE 13.11}

NanoX 200 flexure stage.

The following specifications for this stage are given as:

Unloaded resonant frequency: $700 \mathrm{~Hz}$

Stiffness: $1.1 \mathrm{~N} / \mu \mathrm{m}$

We can use the following formula to calculate the distributed mass moved or "effective mass" of the unloaded structure:

$$
\begin{aligned}
& \qquad m_{\text {eff }}=c_{T} /\left(2 \pi f{ }_{\text {res }}\right)^{2} \\
& m_{\text {eff }}=\text { effective mass } \\
& c_{T}=\text { stiffness } \\
& f^{\circ}{ }_{\text {res }}=\text { unloaded resonant frequency }
\end{aligned}
$$

So for this case we calculate an effective mass of $57 \mathrm{~g}$.

Now we need to move a mirror with a mass of $200 \mathrm{~g}$ and determine the resonant frequency of the system. We can use the following formula:

$$
f_{\text {res }}^{1}=f_{\text {res }}^{0} \frac{\sqrt{m_{\text {eff }}}}{m_{\text {eff }}+\mathrm{M}}
$$

So for this case the resonant frequency has dropped from $700 \mathrm{~Hz}$ to $330 \mathrm{~Hz}$.

Although a good order-of-magnitude estimate, things are actually not quite so simple since the stiffness $c_{T}$ is a variable dependent on the amount of expansion of the stack and the load. Also, off-axis loading may cross couple out-of-plane resonances. When very accurate determinations of resonant frequency are necessary, the best method for determining this is by applying load to the stage under the conditions of use and measuring the response of the structure to a mechanical spike impulse and allowing the structure to "ring" at its resonant frequency.

A simple way to generate a spike mechanical impulse to a stack-flexure structure is by giving the piezo an electrical step function. I loaded this stage with $200 \mathrm{~g}$ and measured the response of the stage with an interferometer (Figure 13.12). A bandpass filter has been applied to the frequency domain graph (Figure 13.13) to isolate the region of interest.

From this you can see the primary resonance is about $300 \mathrm{~Hz}$. Rise times for these types of systems are dependent on the resonant frequency and can be estimated to be about

$$
\frac{1}{3 f_{\text {res }}^{1}} \text { or in this case about } 1 \mathrm{~ms} \text { for the loaded stage. }
$$




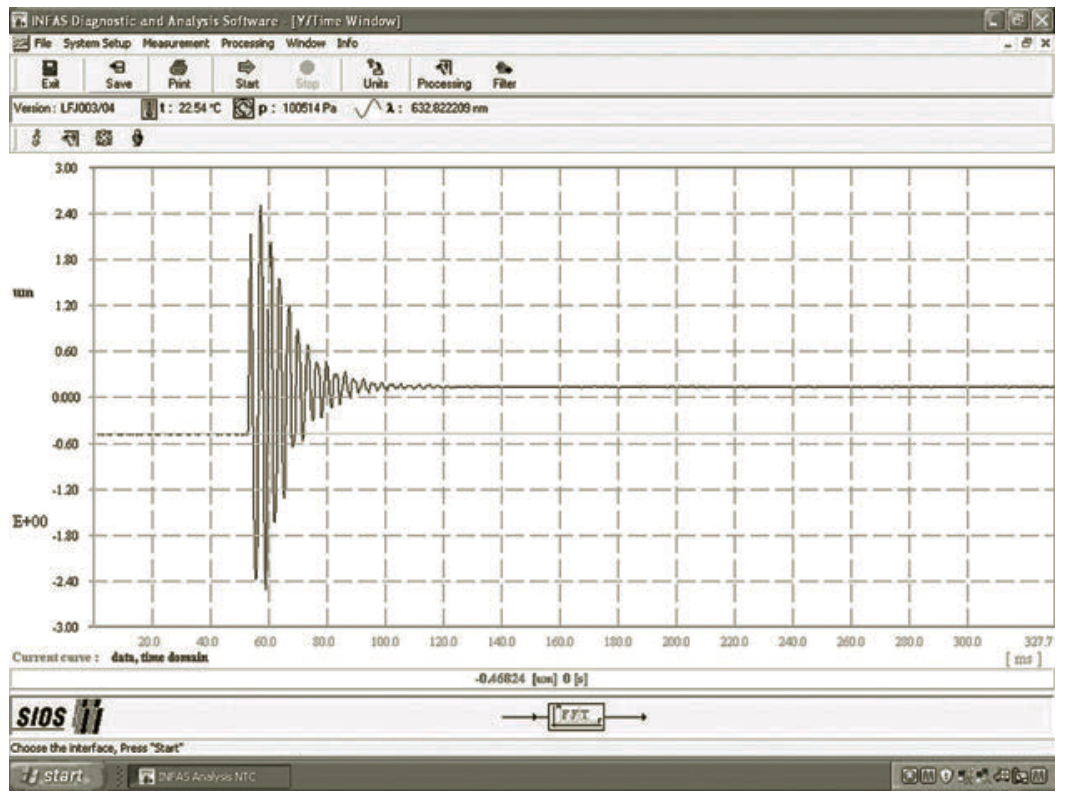

FIGURE 13.12

Step impulse time domain.

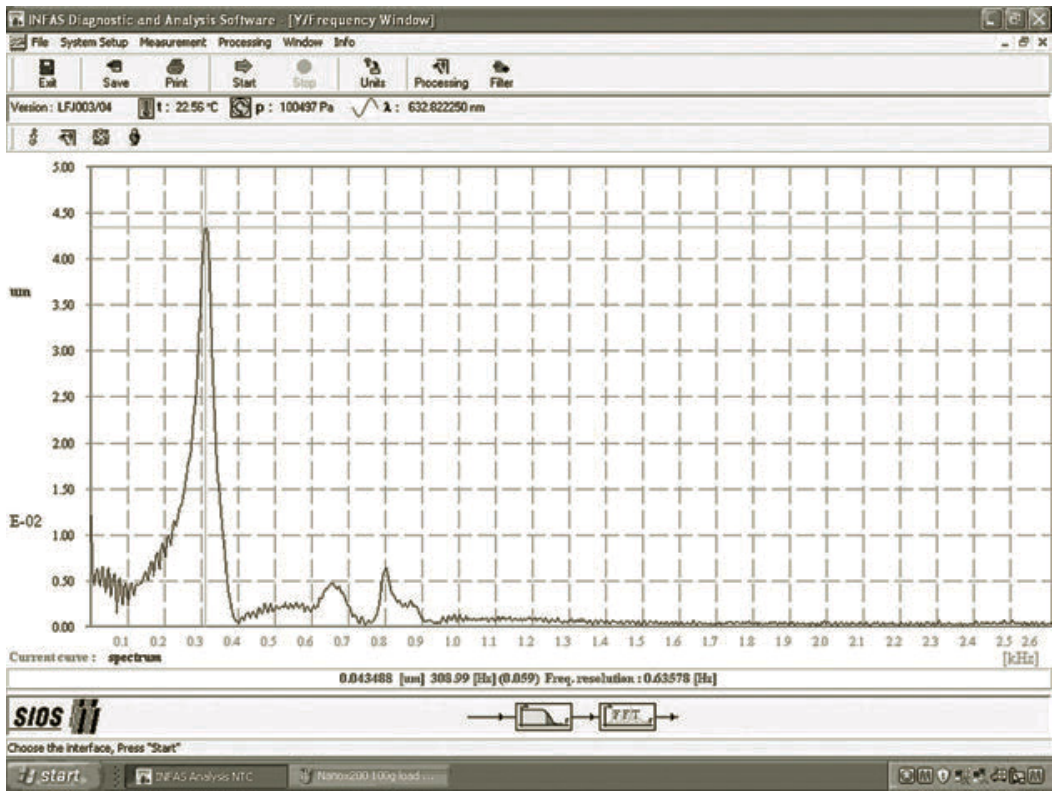

FIGURE 13.13

Step impulse frequency domain. 


\subsection{ELECTRICAL DRIVES}

\subsubsection{Noise}

The positional noise inherent to a piezoelectrical system is only limited by the voltage noise of the power supply that is used to drive it. Commercially available amplifiers built for piezoelectric stages today typically have voltage noise of $<300 \mu \mathrm{V}$ rms over a large bandwidth. Since piezoelectric stacks typically operate over a voltage range of $150 \mathrm{~V}$ this voltage noise can be expressed as a relative noise of $2 \mathrm{E}-6$. So for a piezo stage capable of a total motion of $240 \mu \mathrm{m}$ as in the previous example, we can calculate the rms noise contribution from the electronics as $0.5 \mathrm{~nm}$. It's pretty close to the resolution of the interferometer (0.3 $\mathrm{nm}$ in this case).

\subsubsection{Current}

The main consideration for current requirements of piezo stages is their large capacitance. Formulae for calculating bandwidth and rise times based on available current are simply those used for charging and discharging a capacitor with a very large resistance. So, you can imagine that if you charge a piezo stage to a potential of $70 \mathrm{~V}$ and leave it there it will draw almost no current from the amplifier. In fact, for many static applications a few $\mathrm{mA}$ will be sufficient. Let's consider the case of a single axis tilting stage PSH 4/1 as shown in Figure 13.14.

This stage has a capacitance of $200 \mathrm{nF}$. Let's say we have an amplifier capable of a maximum average output current of $50 \mathrm{~mA}$ and a peak current of $300 \mathrm{~mA}$ and we need to make small adjustments to the position to within $10 \mu \mathrm{rad}$ to steer a laser beam. Based on the current we have, how fast can we do this? We can use the following formula for the time needed to charge or discharge a large capacitor:

$$
d t=C d V / i_{\max }
$$

$$
\begin{aligned}
& d t=\text { change in time } \\
& C=\text { capacitance } \\
& d V=\text { voltage change } \\
& i_{\max }=\text { peak current available from the amplifier }
\end{aligned}
$$

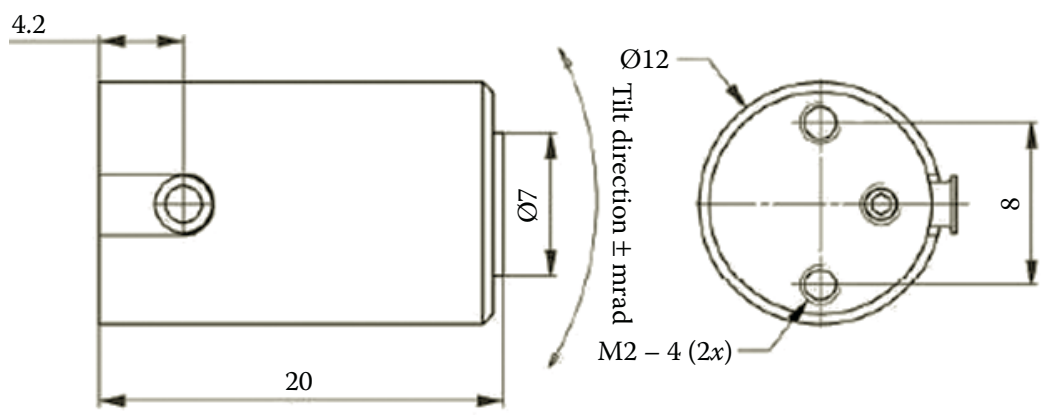

FIGURE 13.14

Small single axes mirror tilt scanning stage. 
For this case we estimate the voltage change as $10 \mu \mathrm{rad} / 4 \mathrm{mrad} \times 150 \mathrm{~V}=375 \mathrm{mV}$ and solving for $d t$ :

$$
\begin{gathered}
d t=(200 \mathrm{E}-9 \mathrm{~F})(375 \mathrm{E}-3 \mathrm{~V}) / 300 \mathrm{E}-3 \mathrm{~A} \\
d t=0.25 \mu \mathrm{s}
\end{gathered}
$$

The limiting factor when considering whether or not to increase the current available to the stage in this type of application should be the mechanical rise time based on the resonant frequency of the system. For this stage the resonant frequency is $6.5 \mathrm{kHz}$. Using the rise time estimation of $d t=1 / 3 f$ we can calculate the maximum rise time limited by the mechanics to $50 \mu \mathrm{s}$. Therefore, increasing our current will not increase the speed of the stage for this application.

Now let's consider the case with a large dynamical requirement. Let's say we would like to scan the same laser beam by tilting the mirror over $+/-1.6 \mathrm{mrad}$ at $4 \mathrm{kHz}$. How much current will we need from our amplifier? For a sinusoidal function we can use the following formula for oscillating a large capacitor:

$$
\begin{aligned}
i_{\max }=\pi f C V p p & \text { peak current required } \\
i_{\text {average }}=f C V p p & \text { average current required }
\end{aligned}
$$

For this case we calculate the Vpp to be $3.2 \mathrm{mrad} / 4 \mathrm{mrad} \times 150 \mathrm{~V}=120 \mathrm{~V}$ and the limiting factor here will be average current available

$$
i_{\text {average }}=(4000 / \mathrm{s})(200 \mathrm{E}-9 \mathrm{~F})(120 \mathrm{~V})=96 \mathrm{~mA}
$$

So we need a bigger amplifier.

These calculations are complicated by the fact that the capacitance of the stacks may increase by up to $200 \%$ due to changes in amplitude, strain, and temperature. So, for this case an amplifier with $200 \mathrm{~mA}$ should be sufficient under a variety of environmental conditions.

Some mention should be made here that driving piezo stacks with very large currents ( $>1$ A) may result in heating of the stack due to the electrical power requirements. When the stack reaches its Curie temperature (around $170 \mathrm{C}$ ) it will depolarize and stop working. Once it cools, you can usually repolarize the crystal by cycling it a few times from 0 to 150 V. Generally speaking I wouldn't recommend this type of high-temperature operation for extended periods of time since it affects the lifetime of the PZT stack.

\subsection{RELIABILITY}

Stack-flexure piezo stages have no parts that move against each other. As friction-free systems, the mechanics exhibit no wear or fatigue as long as the deformation of the metal is kept below its elastic limit. Commercial stages have been operational for more than 15 years of continuous use. The main mechanism of failure for a piezo stage is ion migration of the electrodes into the PZT which eventually causes a short circuit of the stack. This 
effect is accelerated by three conditions which should be avoided if possible:

1. Constantly applied high voltage

2. High-humidity conditions

3. High-temperature conditions

I could put a graph in here showing MTBF for these conditions, but in my experience handling and sealing play a big role in the environmental factors and cycling away from 150 $\mathrm{V}$ a few times a day will do wonders for piezo health and well-being.

So be forewarned if you want to park your stack with greasy fingers at $150 \mathrm{~V}$ for weeks on end in the jungle!

\subsection{TILTING STAGE DESIGN}

There are a few varieties of tilt and tip-tilt stages commercially available. Usually they are temperature compensated for tilt, but not $z$. Since these are mainly used for laser scanning applications and are usually in direct competition with galvo type scanners, the design emphasis here is on high resonant frequency and stiffness. Often flexure hinges are dispensed with in the interest of speed. Three or four piezos are directly preloaded against a top plate and either act independently or in a push-pull configuration. For the three piezostack version, two tilting axes are arranged orthogonally to each other, while an optional third axis is oriented at $45^{\circ}$. A diagram of such a stage is shown in Figure 13.15.

$X, Y$, and $Z$ shown as dashed lines above represent the tilting axes when the individual PZT stacks are actuated in the housing. When all three are given an equal applied voltage, the top plate will move in the $z$ direction. Temperature changes will move the stage in $z$, but not affect the tilting angle. This particular stage design may offer tilting of 1 to $4 \mathrm{mrad}$ with resonant frequencies in the $\mathrm{kHz}$ range. Disadvantages of this design are the location of the pivot point and the fact that it does not offer plus-minus tilting-each axis will tilt in only one direction.

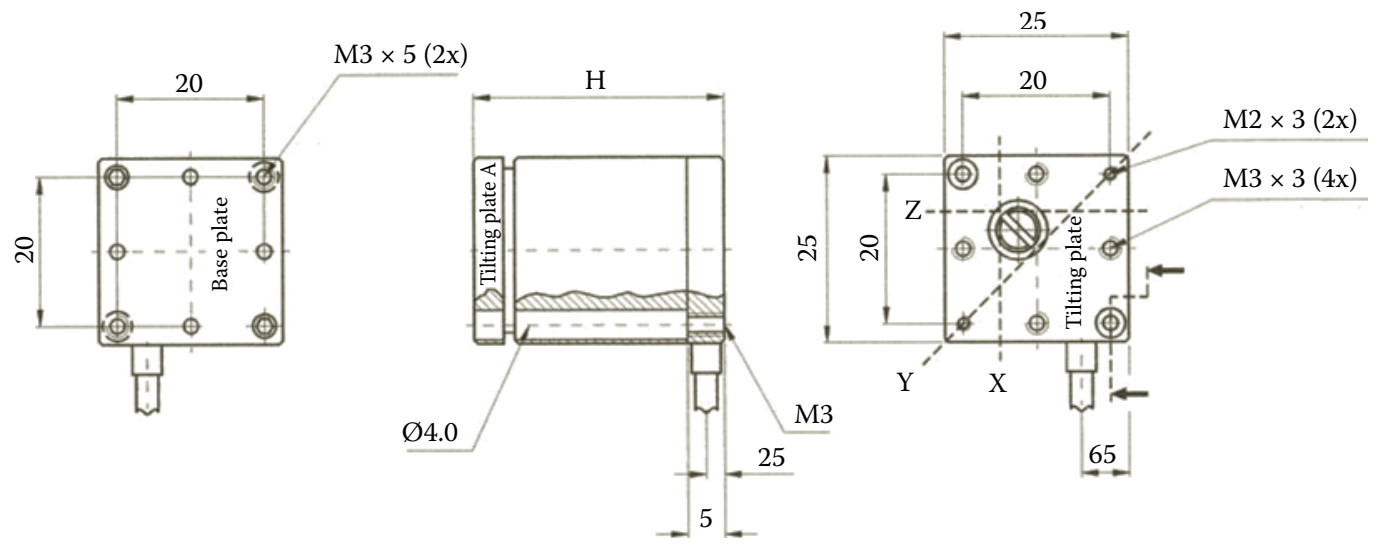

FIGURE 13.15

Three-axis mirror tilt scanning stage. 
(a)

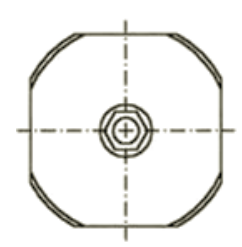

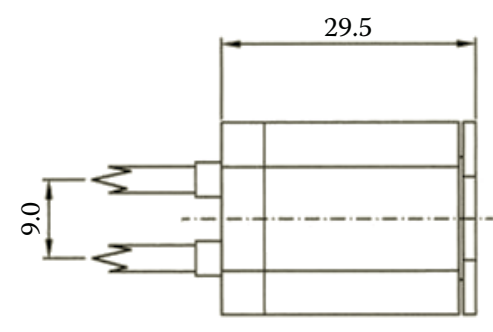

(b)

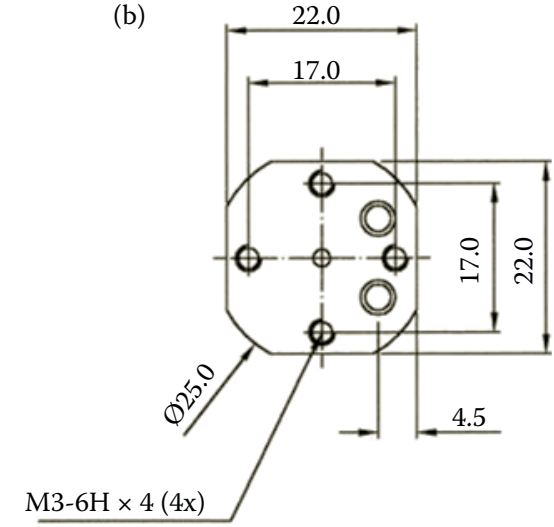

\section{FIGURE 13.16}

Two-axis mirror tilt scanning stage with push-pull design: (a) top (b) bottom.

When a tip-tilt stage with a central pivot point with plus-minus tilting is desired, a four stack push-pull approach is necessary. Once again, the stacks act directly against the top plate in order to maintain a high stiffness and high resonant frequency for the design. A diagram of such a stage is shown in Figure 13.16.

A larger tilting angle is possible with this push-pull approach and these stages are capable of up to $10 \mathrm{mrad}(+/-5 \mathrm{mrad})$ of tilt angle with resonant frequencies in the $\mathrm{kHz}$ range.

\subsection{LINEAR STAGE DESIGN}

Today there is a demand for nanopositioners capable of a variety of specialized tasks. This wide variety of applications has resulted in a number of innovative new designs based on flexure techniques. Powerful computer algorithms utilizing FEA optimization have resulted in systems specialized for high resonant frequency under load, and minimized tilting and cross-talk errors over relatively large motions (large for a piezo stage is a $\mathrm{mm}$ ).

\subsubsection{Cross talk}

Single dimension flexure-stack translation stages use a parallel guidance design. They use flexing monolithic solid metal hinges to guide and amplify the motion of the stack they contain. Usually the metal structure is designed so that they must be flexed slightly prior to mounting the piezo into them to provide an integrated preload and an epoxy is used to permanently bond the stack inside. A diagram and picture showing the design of such a structure and its associated motion are shown in Figures 13.17 and 13.18.

The motion in the diagram on the left has been exaggerated so that we can clearly see the problem here; in addition to the motion in the desired direction, we also have two other types of cross talk. There is a lateral component and a rotational component. A typical asymmetrical piezo flexure design such as this may have lateral cross talk on the order of $200 \mathrm{~nm}$ for a $100-\mu \mathrm{m}$ stage. All four flexure hinges in the parallelogram design show this 


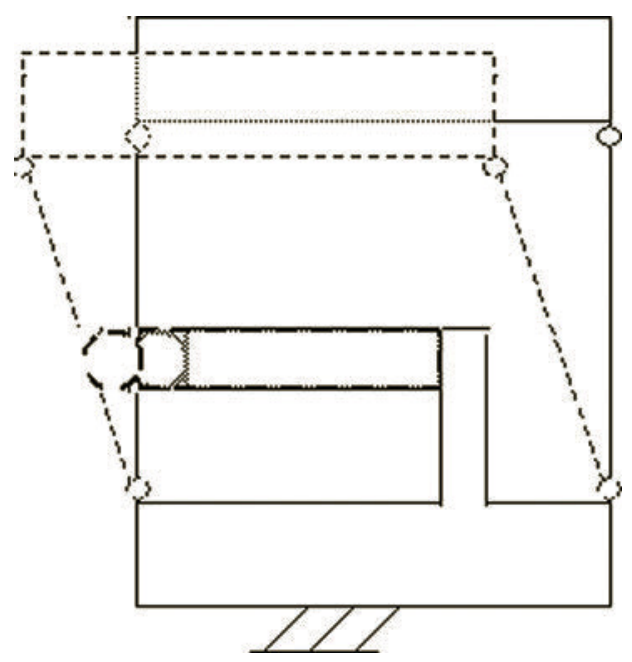

FIGURE 13.17

Guidance diagram for parallelogram stage.

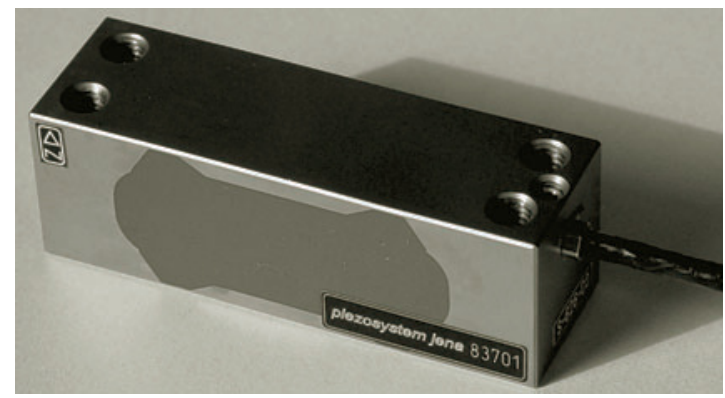

FIGURE 13.18

Picture of a parallelogram stage F.

phenomenon. This is caused by a parallel deviation which is exerted around a rotational center. Also, as mentioned previously, the stacks themselves exhibit motion vectors in all six degrees of freedom. Although the flexure hinges direct these force vectors through very small areas, thus minimizing the impact of the unwanted motion, some forces from the stack operate outside these stress areas and contribute to additional cross talk not inherent in the design. Also a residual mechanical asymmetry caused by asymmetrical stack point of contact contributes and so the total rotational cross talk component is typically $15 \mu \mathrm{rad}$ for $100 \mu \mathrm{m}$ of motion. The major advantage of such a simple four hinge parallelogram guidance design is the low inertia in combination with a suitable guidance behavior; it is well suited for fast nanoscanning applications.

\subsubsection{Minimizing Cross talk}

It is possible to minimize this stack-induced cross talk by carefully selecting well-behaved stacks and actively aligning them with an interferometer prior to permanent epoxy bonding. This might reduce this type of cross talk by one-third, but cannot eliminate it altogether and as you might imagine, is time consuming and expensive. 
By applying meander ordered hinges we obtain an elegant solution to the problem of cross talk by allowing the hinges to compensate each other. Such a system is shown in Figure 13.19.

Of course there are tradeoffs here for improved performance. In this case, the number of flexure hinges has been increased from 4 to 16 . This significantly weakens the mechanical structure of the stage. Stiffness has now dropped dramatically and therefore results in significant reductions in resonant frequency for any laterally applied load to the system. Reduced resonant frequency means higher noise characteristics since a lower resonant frequency is more easily excited by ambient noise. However, for applications requiring highly accurate nanopositioning with light loads this solution works well and is free of parasitic cross talk.

\subsubsection{Increasing stiffness}

So how can we obtain the benefits of a symmetrical dual flexure structure and maintain our excellent noise properties?

One weakness of parallelogram flexure structure is that piezo stack provides a strong compressive force and stiffness, but we rely on the weak spring memory forces of the metal for our reset forces. One way to increase the reset forces of the flexure structure would be to increase the thickness, for example, reset force of the flexure hinge. This has the effect of making the entire structure more stiff and, unfortunately, since the desired DOF is blocked this increases parasitic rotational errors. Another way to increase the stiffness of the stage is to apply a push-pull concept to the design. We integrate two stack actuators into a flexure structure in such a way that they act in concert with each other and provide both compressive and reset forces to the structure for the motion of the stage. For this design concept we can increase stiffness by up to ten times in the intended direction of motion and by up to two times for the whole system. Additionally the overdetermination of this design results in a high degree of inner preload that makes the stage much more robust against mishandling, off-center loads and high loads. All featured without increasing rotational error as per Figure 13.20.

A main advantage of such a push-pull concept is the separation of guidance and gearing. As opposed to traditional designs, the flexure hinges are exclusively responsible for trajectory tasks; they are not responsible for exerting reset forces. Also, the stacks, when

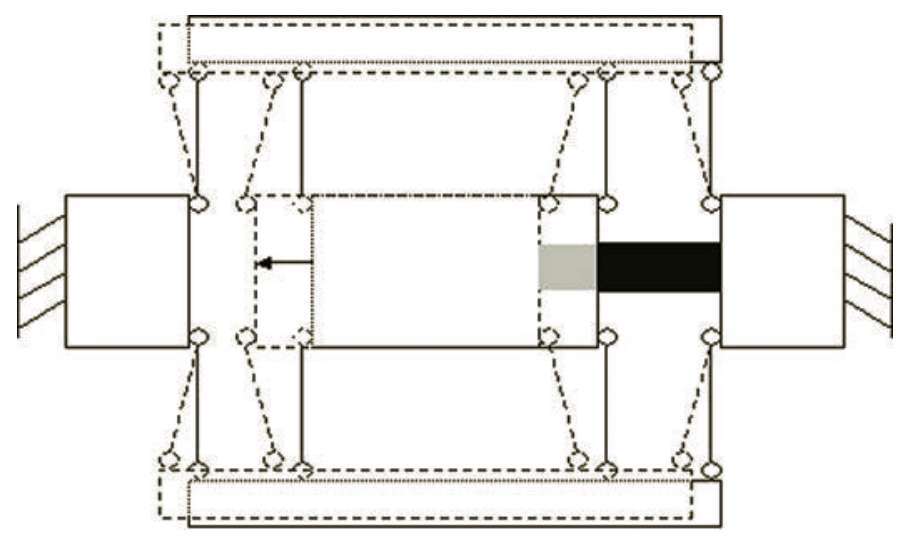

FIGURE 13.19

Guidance diagram using meander ordered hinges. 


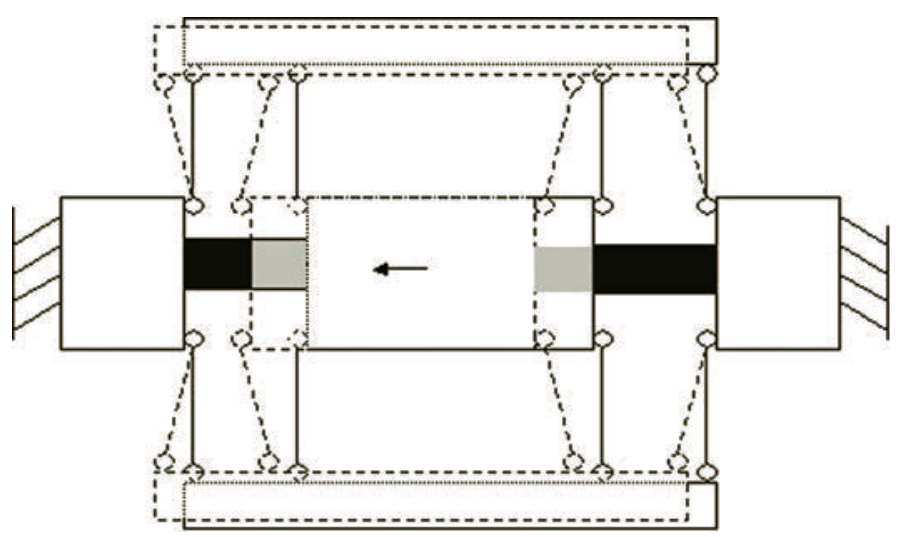

FIGURE 13.20

Guidance diagram of a push-pull drive mechanism.

arranged in alternating order, are able to accelerate and stop actively taking advantage of their huge pressure force potential. So this design offers a maximum of dynamical and guidance behavior. It is inherently temperature compensated and well suited for nanopositioning tasks as well as nanoscanning applications.

\subsection{DAMPING}

You can abstract a piezoactuator as a linear oscillating system consisting of spring, damper, and inertial mass, following the normalized mathematical description (force equilibration) (Figures 13.21 and 13.22):

$$
k x+c \frac{d x}{d t}+m \frac{d^{2} x}{d t^{2}}=F_{d}(t)=1
$$

$$
\begin{aligned}
k & =\text { spring constant } \\
x & =\text { stroke } \\
c & =\text { damping coefficient } \\
\frac{d x}{d t} & =\stackrel{\circ}{x}=\text { velocity } \\
m & =\text { inertial mass } \\
\frac{d^{2} x}{d t^{2}} & =\stackrel{\infty}{x}=\text { acceleration } \\
F_{d} & =\text { disturbance (force) } \\
t & =\text { time }
\end{aligned}
$$

Solving the differential equation in respect to the initial values

$$
x(t=0)=0
$$

and

$$
x(t=0)=0
$$




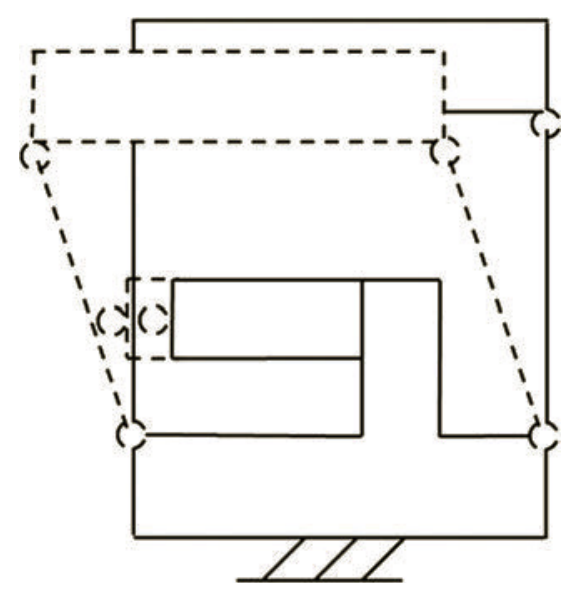

FIGURE 13.21

Schematic piezoactuator.

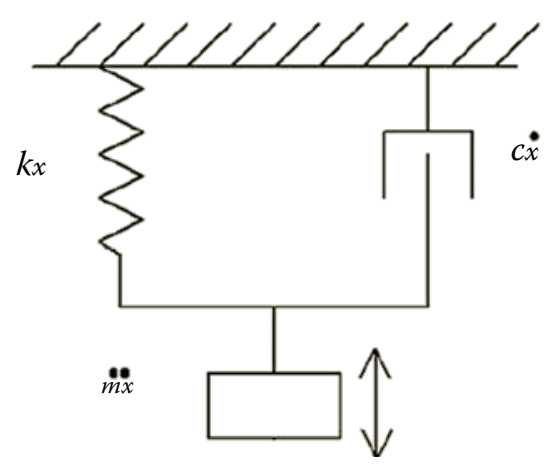

\section{FIGURE 13.22}

Analytic abstraction.

you obtain the response of the oscillating system to the external disturbing force (Figures 13.23 and 13.24). Depending on the damping ratio the behavior is described as:

$\begin{array}{ll}\text { undamped } & D=0, \\ \text { underdamped } & 0<D<1, \\ \text { critically damped } & D=1, \\ \text { overdamped } & D>1\end{array}$

with $D=\frac{c}{2 \sqrt{k m}}$

$D=$ damping ratio

An increasing damping ratio decreases the resonance magnification and sharpness. That means the resonance appears at a lower frequency but its suppression is much higher. ${ }^{1-3}$

Because of the marginal electrical losses of the piezoceramic that could reduce kinetic energy from the system by transforming into heat, in practice the underdamped piezoactuator is the usual one (Figure 13.25). The transient oscillation is described as

$$
x(t)=1-e^{-D w_{0} t}\left[\cos w t+\frac{D w_{0}}{w} \sin w t\right]
$$




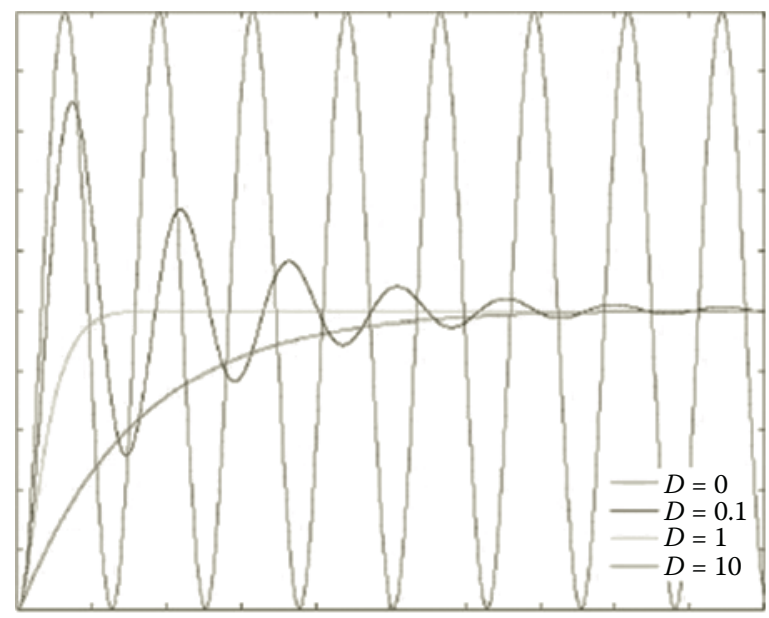

FIGURE 13.23

Disturbance response-time domain (amplitude vs. time) showing undamped, underdamped, critical damped, overdamped. The fastest is the critical damped case.

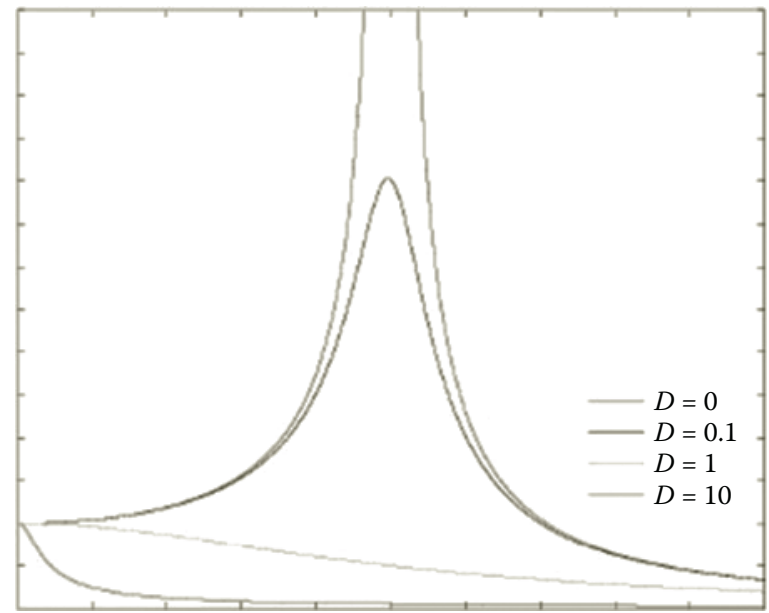

FIGURE 13.24

Disturbance response-frequency domain (magnitude vs. frequency) showing undamped, underdamped, critical damped, overdamped. Listed largest to smallest amplitude.

with $\quad w=w_{0} \sqrt{1-D^{2}}$

$\omega=$ underdamped frequency

$\omega_{0}=$ eigenfrequency (w/o damping)

and $x_{\text {envelope }}(t)=1 \pm e^{-w_{0} D t}$

$x_{\text {envelope }}=$ envelope

describes the turning points (envelope). ${ }^{1,3,4}$

As said the damping behavior of a piezoactuator is marginal, well suited for ultrafast scanning applications and small loads. However, fast high load applications using the proprietary outstanding stiffness of the piezoceramic can be optimized by implementation of fitted passive viscoelastic damping parts. 


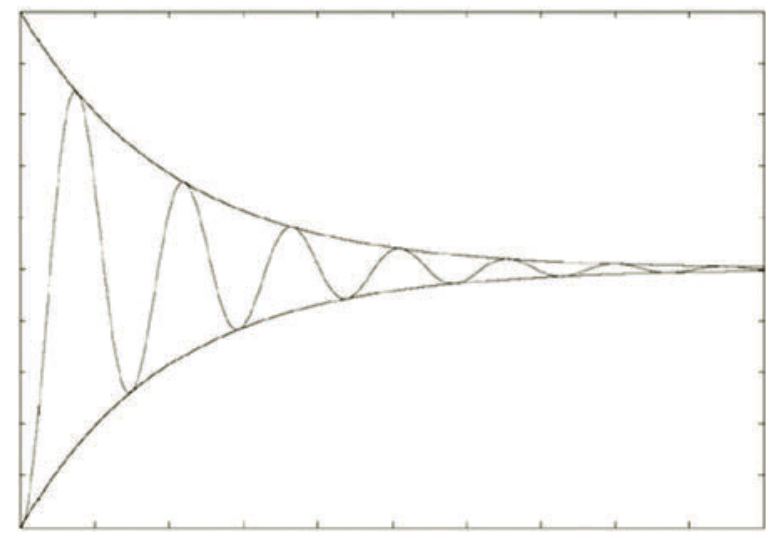

FIGURE 13.25

Amplitude vs. time graph: underdamped oscillation and its envelope.

The critical damping (aperiodic case) shows the shortest settling time and no overshooting, but the damping ratio depends on the inertial mass. Therefore you are able to optimize for one load configuration only. Higher loads will settle much faster than in the undamped case otherwise small load scenarios will behave unnecessarily slow. So it's recommended for versatile and robust use that the stage works in the underdamped range, close to the critically damped case.

But, how do you determine a well damped setup? For our assessment we use the damping ratio, that you can determine in such a way:

1. First, you generate a rectangular wave. Due to the fact that such a waveform consists of odd-numbered multiples of the fundamental frequency, the piezoactuator's resonances will be excited according to the following formula

$$
x_{r}(t)=\frac{4 h}{p} \sum_{k=1}^{\infty} \frac{\sin \left[(2 k-1) w_{f} t\right]}{2 k-1} ; \quad k=1 . . \infty ;
$$

$x_{r}=$ current stroke of rectangular wave

$h=$ degree of amplitude

$v_{f}=$ fundamental frequency, depending on the bandwidth of the function generator and amplifier

2. Next, you modulate the amplifier output signal and the piezoactuator oscillates in its resonances.

3. Transforming the oscillation into a frequency spectrum via, for example, fast Fourier transformation (FFT) allows you to determine the damping ratio per for example bandwidth method (Figure 13.26).

As mentioned before damping ratio and resonance sharpness correlate to each other. So you can calculate the damping ratio using the equation

$$
D=\frac{f_{2}-f_{1}}{2 f_{r}}
$$



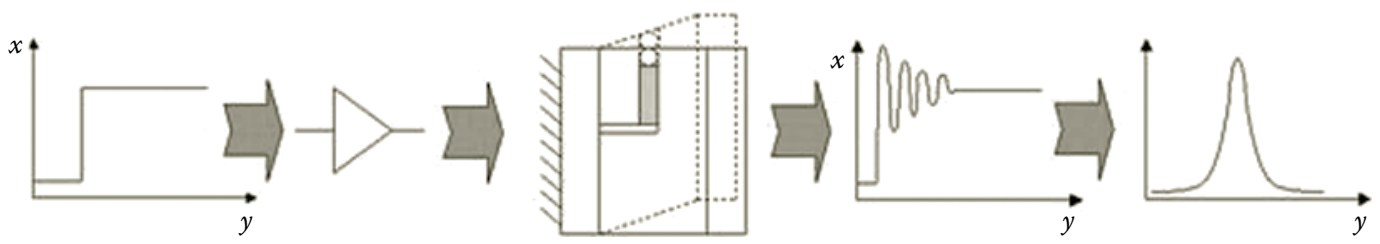

FIGURE 13.26

Scheme of determining the damping ratio of a piezoactuator.

$$
\begin{aligned}
& f_{1}=\text { lower frequency @ } m_{\max } / \sqrt{2} \\
& f_{1}=\text { upper frequency @ } m_{\max } / \sqrt{2} \\
& f_{r}=\text { resonant frequency } \\
& m_{\max }=\text { resonant magnitude }
\end{aligned}
$$

The longer the measured time (real or zero padded), the higher the frequency resolution and more accurate the resulting damping ratio calculation (Figures 13.27 through 13.30). Following the sample theorem (SHANNON/NYQUIST), the sample frequency has to be at least two times of the frequency that is to be measured. ${ }^{4}$

Using viscoelastic damping in open loop order offers a lot of advantages. Firstly, the settling time decreases significantly. The piezoactuator reacts much more robustly against high loads and dynamic forces, as well as mishandling and environmental oscillations. Also, the positioning noise caused by the noise of the amplifier is deeply suppressed. So you can perform subnanometer step scans in much smaller stepwidth, even at higher loads. However, the viscoelastic and piezoelectric intrinsic drift accumulates. Using viscoelastic damping in closed loop, the drift doesn't exist. ${ }^{5,6}$

\subsection{CLOSED LOOP SYSTEMS}

We've discussed a number of problems inherent to piezo technology for motion control. These include drift or creep, hysteresis, temperature dependence, nonlinear expansion, variance among individual stacks, cross talk, and rotational error. The simplest way to address many of these simultaneously is to characterize the piezosystem with an integrated measurement device and integrate a closed loop feedback mechanism to control the position. In order to take advantage of the piezosystem's capability for high resolution in the nm range and high dynamic capabilities it is important to select a type of sensor which is fast with nanometer accuracy. Clear choices that satisfy these requirements are capacitive sensors, strain gage sensors, inductive (LVDT) sensors, optical scales, and interferometric measurements. In order to somewhat limit the scope of the chapter we will concentrate on the use of capacitive sensors and strain gages in PID closed loop systems since these will be useful for a majority of applications which use piezos.

\subsection{STRAIN GAGES}

Strain gages can be applied directly onto a stack of PZT to measure strain that has been produced by the expansion of the PZT or onto the flexing hinges of the flexure structure. 


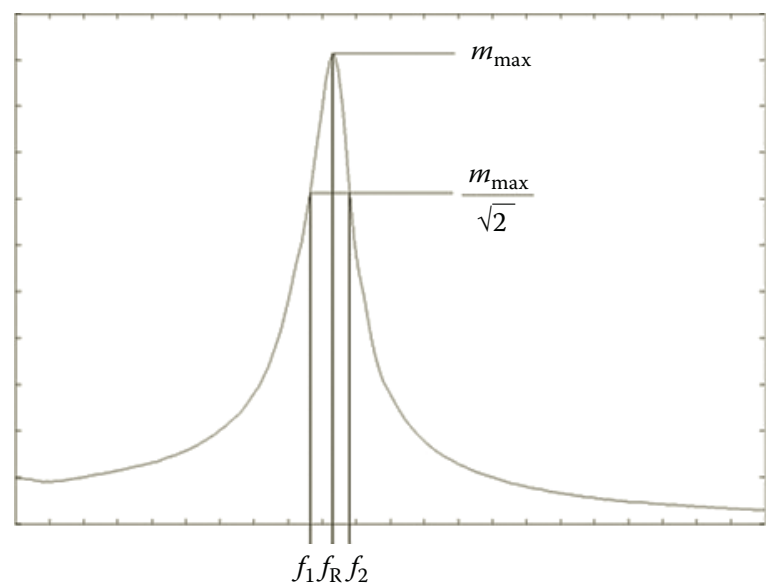

FIGURE 13.27

Magnitude versus frequency graph: determination of damping ratio via bandwidthmethod.

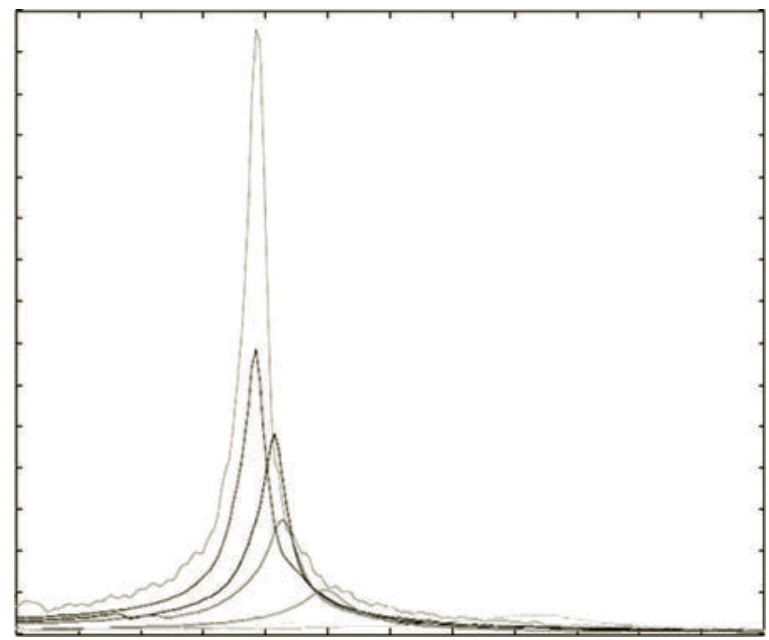

FIGURE 13.28

Disturbance response-time domain (amplitude vs. time): increasing damping ratio (left to right).

Since the expansion and therefore strain of a PZT stack are inhomogeneous and the strain gage is applied to just a small part of a stack, it is much more accurate (usually at least $2 \times$ ) to apply the strain gage to the flexure hinges-usually more than one. This effectively makes a measurement summation of all motion "prior" to the flex hinge, while ignoring any motion which occurs "after" the hinge. Therefore all PZT motions including temperaturedependent ones are measured, but the strain gage system will not compensate for expansion of the top plate of a stage. Special care must be taken for a proper bond to the PZT or metal so that adequate heat transfer occurs to minimize Johnson-Nyquist noise since strain gages are resistive devices. A variety of strain gages are commercially available which are specifically tailored to various material expansion coefficients and the proper gage must be selected for the stage material (i.e., steel, aluminum, INVAR). A typical strain gage is either a full or half wheatstone bridge with $5 \mathrm{KOhm}$ resistors arranged as in Figure 13.31. 


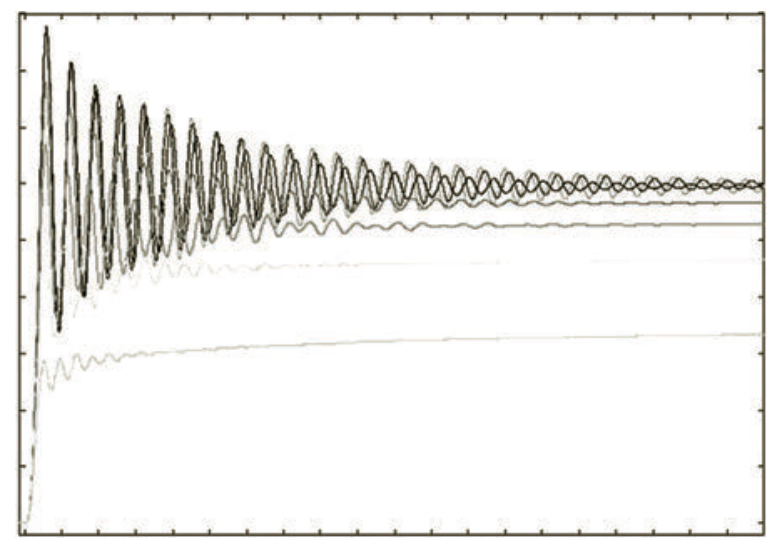

FIGURE 13.29

Disturbance response in open loop-time domain (amplitude vs. time): increasing damping ratio (top to bottom).

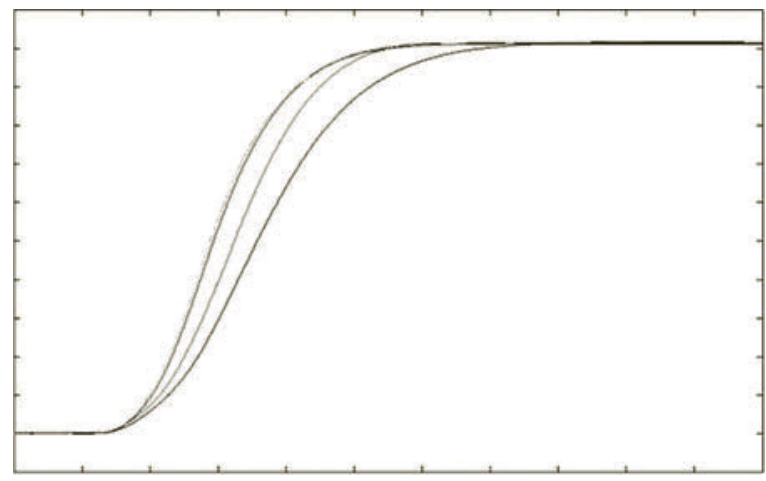

FIGURE 13.30

Disturbance response in closed loop-time domain (amplitude vs. time): increasing damping ratio (right to left).

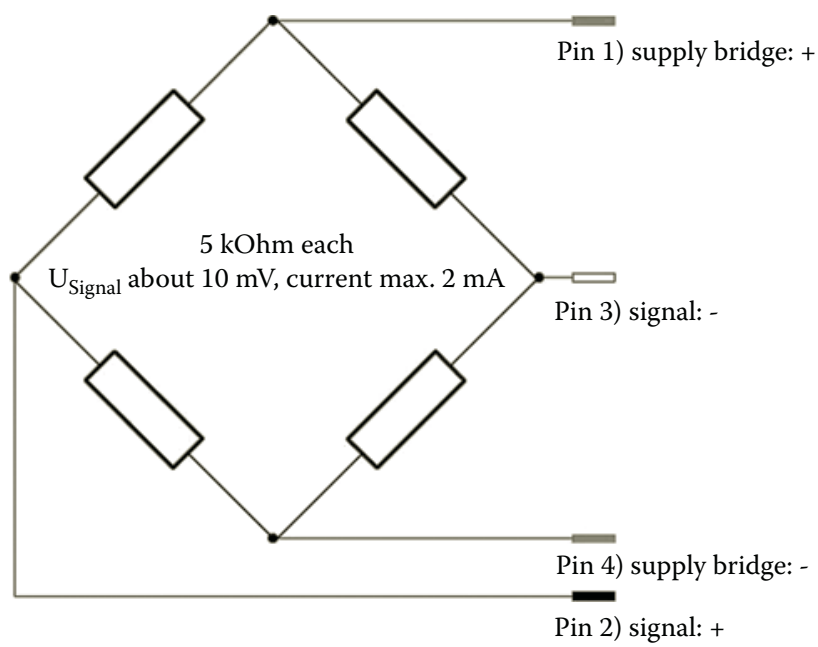

FIGURE 13.31

Electrical diagram of a strain gage. 


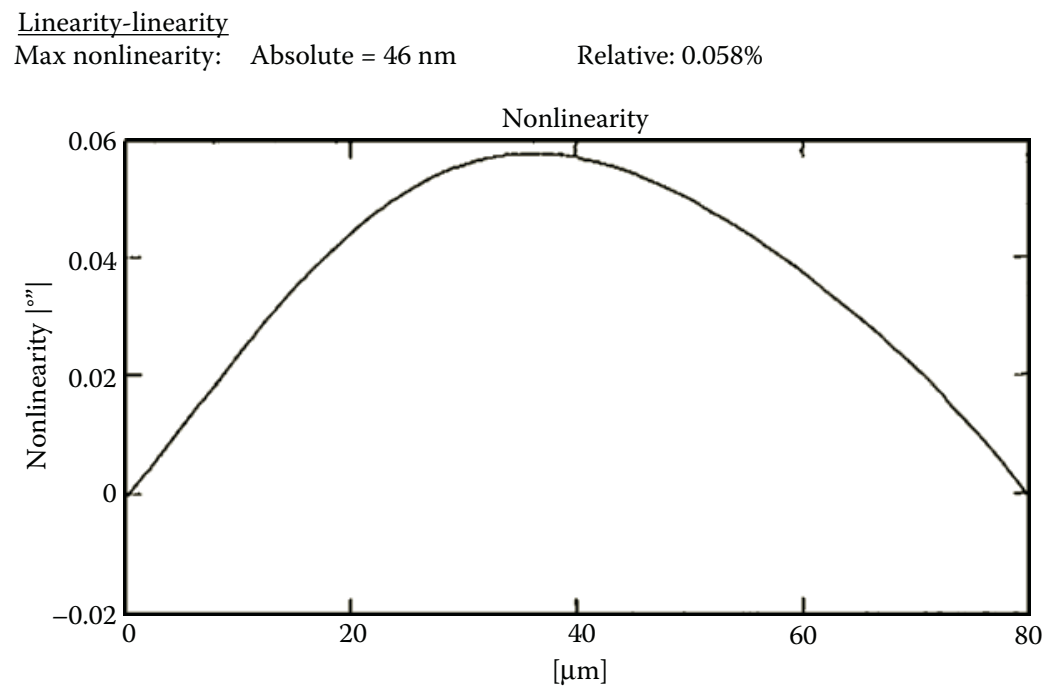

FIGURE 13.32

Nonlinearity of a strain gage sensor.

With a full wheatstone bridge expansion or contraction due to temperature variations do not affect the output signal of the sensor since all resistors will be affected equally. With a half-wheatstone bridge, expansion or contraction due to temperature variations will be measurable. I've run into very few situations where a half bridge is desirable. It's important to make this distinction since both designs are used for closed loop feedback in commercially available piezosystems and you should be aware of the type of system you're using. The typical safe bending radius for such a gage is about $1.5 \mathrm{~mm}$ which is far above the elastic limit of steel. Often a flexure can be damaged by applying excessive force and, as a result, it is bent beyond the elastic limit of the metal such that the strain gage still functions, but is permanently deformed so that the strain gage shows some permanent DC offset. In some cases it is possible to repair the stage by measuring the output of the strain gage while bending the flexure back until the DC offset is minimized and then performing a recalibration of the closed loop system. The response of a strain gage to motion is fairly linear. Figure 13.32 shows the voltage response of a strain gage when a flexure is actuated over a distance of $80 \mu \mathrm{m}$. The distance traveled was measured with an interferometer.

Often, nonlinearity curves follow parabolic functions and it is a fairly common practice to correct for these errors using polynomial fit algorithms or simple look-up tables.

\subsection{CAPACITIVE SENSORS}

Capacitive sensors use a charged flat metal plate of precisely determined area to make a noncontact measurement of distance based on formula for parallel plate capacitors. Although it sounds simple in theory, in practice this type of measurement can be quite complex due to the very small changes in capacitance that must be measured in order to achieve nm resolutions. Complex electronics must be modulated at specific frequencies 
for various bandwidths and resolutions that are desired. Since changes in capacitances being measured are nearly on the same order of magnitude as cable capacitances, specially calibrated cables and connectors must be used. It is important to keep this in mind when selecting a capacitive measurement system for closed loop. Often it is not possible to change cable lengths or connectors for your system if the capacitive sensor electronics are built directly into the closed loop amplifier system. On the plus side, capacitive sensors offer the following advantages over strain gage systems: lower noise, higher bandwidth capability, and better linearity. Also, it is possible to arrange capacitive sensors such that they measure directly on the point of interest for the stage-or at least closer to it. Cross talk and temperature-dependent motion not measured by a strain gage are now measurable and correctable by a capacitive measurement system.

\subsection{ELECTRONIC CONTROL ARCHITECTURE FOR CLOSED LOOP SYSTEMS}

A simple diagram of a typical closed loop system is as shown in Figure 13.33.

Closed loop systems can be made completely analog, digital, or as a hybrid analogdigital system (for instance an analog PID control system with digital potentiometers for programmability).

Variables which must be calibrated based on the individual system are as follows:

1. Gain of the sensor

2. Offset of the sensor

3. Gain of the measurement interfaces (e.g., 0-10 V analog output, digital display, A/D interface for serial port)

4. Total travel of the system over the full voltage range (gain of the positioning system)

5. Gain parameters of the P, I, and D components of the system

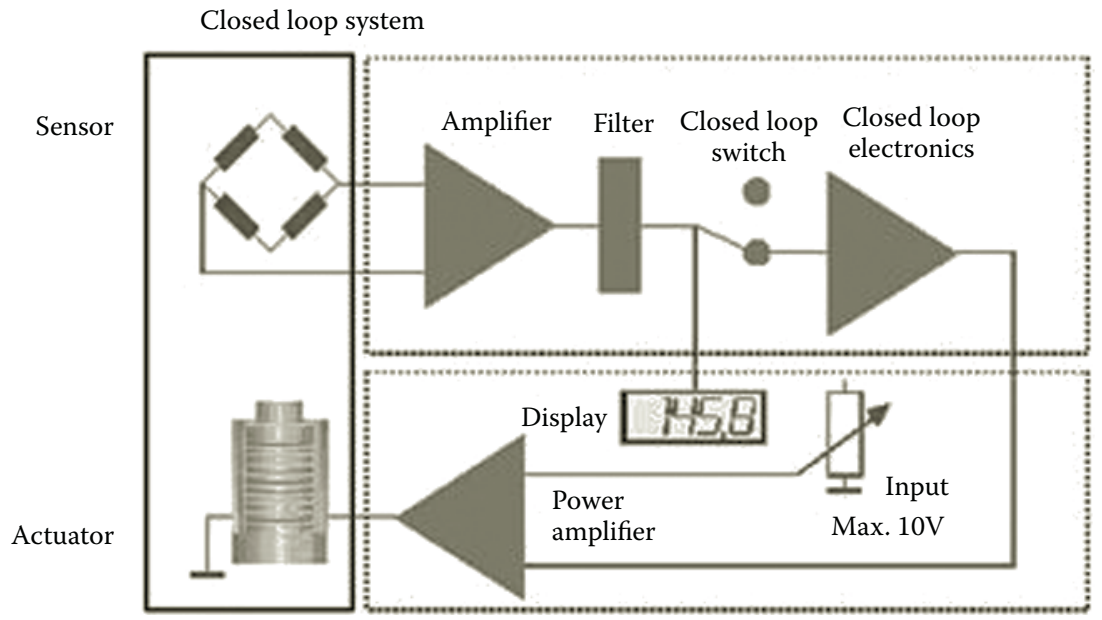

FIGURE 13.33

Closed loop system architecture. 
Let's take the example of a stage that specifies its total open loop travel as $100 \mu \mathrm{m}$ under light load conditions. Since, as mentioned previously, PZT stacks can vary quite a bit the actual motion from such a stage could vary as much as $20 \mu \mathrm{m}$. For this example let's assume the stage is capable of $110 \mu \mathrm{m}$ for a voltage range of $-10 \mathrm{~V}$ to $150 \mathrm{~V}$. This intrinsic gain of the system will determine a number of factors in the final closed loop system since linearity, resolution, and repeatability are some function of the total motion amplification. Often, manufacturers will guarantee and characterize their system specifications as some percentage of total measured motion of the system in closed loop (i.e., a resolution of $0.05 \%$ for this system in closed loop would be $4 \mathrm{~nm}$ ) even though it may actually be determined by the total motion the system is capable of making in open loop. In other words, taking a $400 \mu \mathrm{m}$ stage and calibrating it for $80 \mu \mathrm{m}$ of motion in closed loop instead of $360 \mu \mathrm{m}$ will not increase the resolution of your system.

As we saw before, a piezosystem will overshoot and oscillate at some resonant frequency. Closed loop systems have the job of damping and controlling this behavior, but some overshoot and settling time, especially for step functions, is unavoidable. In order to allow for this behavior, closed loop systems are calibrated within a range of $80 \%$ of the full motion allowing $10 \%$ at the extremes of the travel range for overshooting (Figure 13.34). Since piezos vary from system to system this range is often further reduced to allow for PZT stacks that may have less travel than others. For this particular case a travel of $80 \mu \mathrm{m}$ would be calibrated into the system since the minimum specified travel range is $100 \mu \mathrm{m}$.

The speed of a closed loop system is determined by the loading and resonant frequency of the system. Introducing a high load to a system calibrated for light loads may cause it to become underdamped and it may begin to oscillate. The PID can be adjusted in such cases to damp out the oscillation, but the rise time will suffer as a result. In some cases noise due to oscillation of an underdamped system is tolerated in the interest of speed. As an example, a stage which moves $100 \mu \mathrm{m}$ in open loop calibrated for $80 \mu \mathrm{m}$ in closed loop is loaded with a microscope objective which weighs $300 \mathrm{~g}$. At rest the PID closed loop begins to oscillate at a frequency of about $130 \mathrm{~Hz}$ as shown in Figure 13.35.

Rise time for the system is about $11 \mathrm{~ms}$ for a step of $0.6 \mu \mathrm{m}$ and P_P noise is about $6 \mathrm{~nm}$. We can eliminate the oscillation by overdamping the system with the PID closed loop with results as in Figure 13.36.

As you can see the problem with oscillation has been reduced by a factor of 3 , but the settling time to a final position has been extended from $11 \mathrm{~ms}$ up to about 25-30 ms.

Final tuning of a closed loop system is specific to the particular application. In this case, which is optical microscopy, the depth of field of this particular microscope objective was well in excess of the $6 \mathrm{~nm}$ and settling time was of primary importance. Another

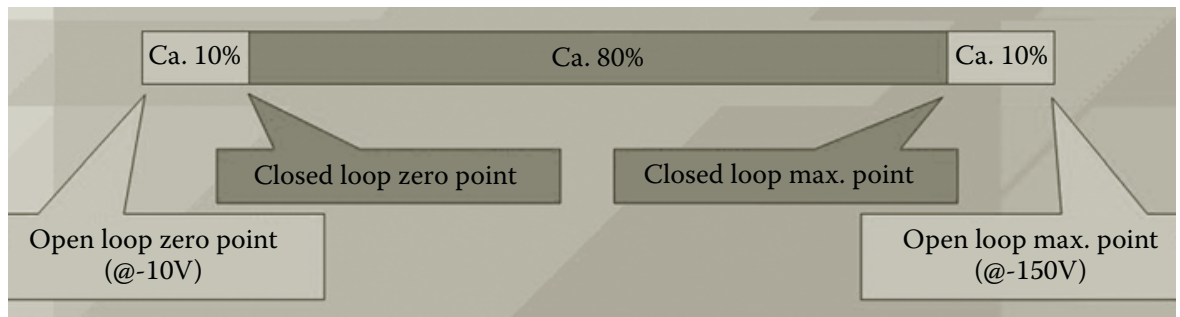

FIGURE 13.34

Closed loop versus open loop travel range of a piezo stage. 


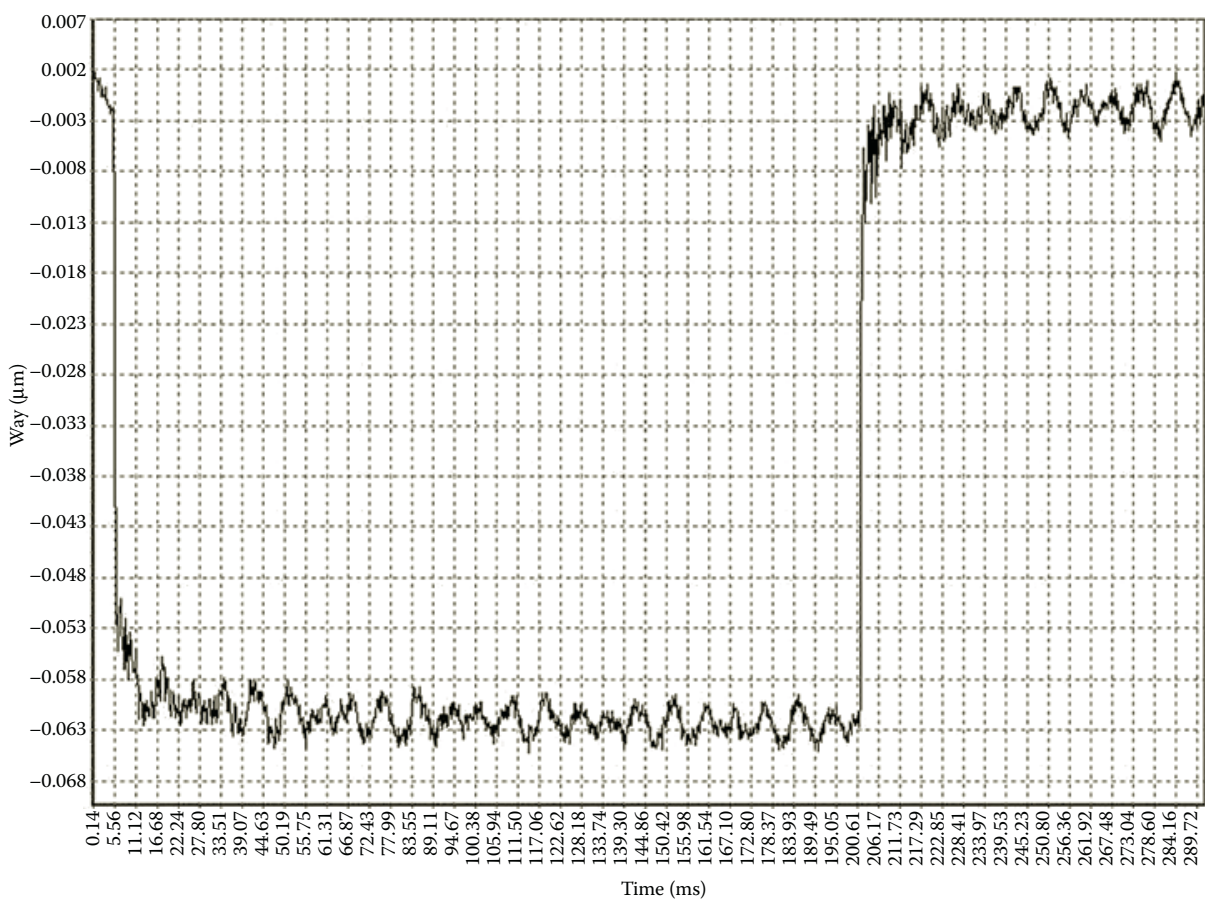

\section{FIGURE 13.35}

Closed loop system subject to additional loading.

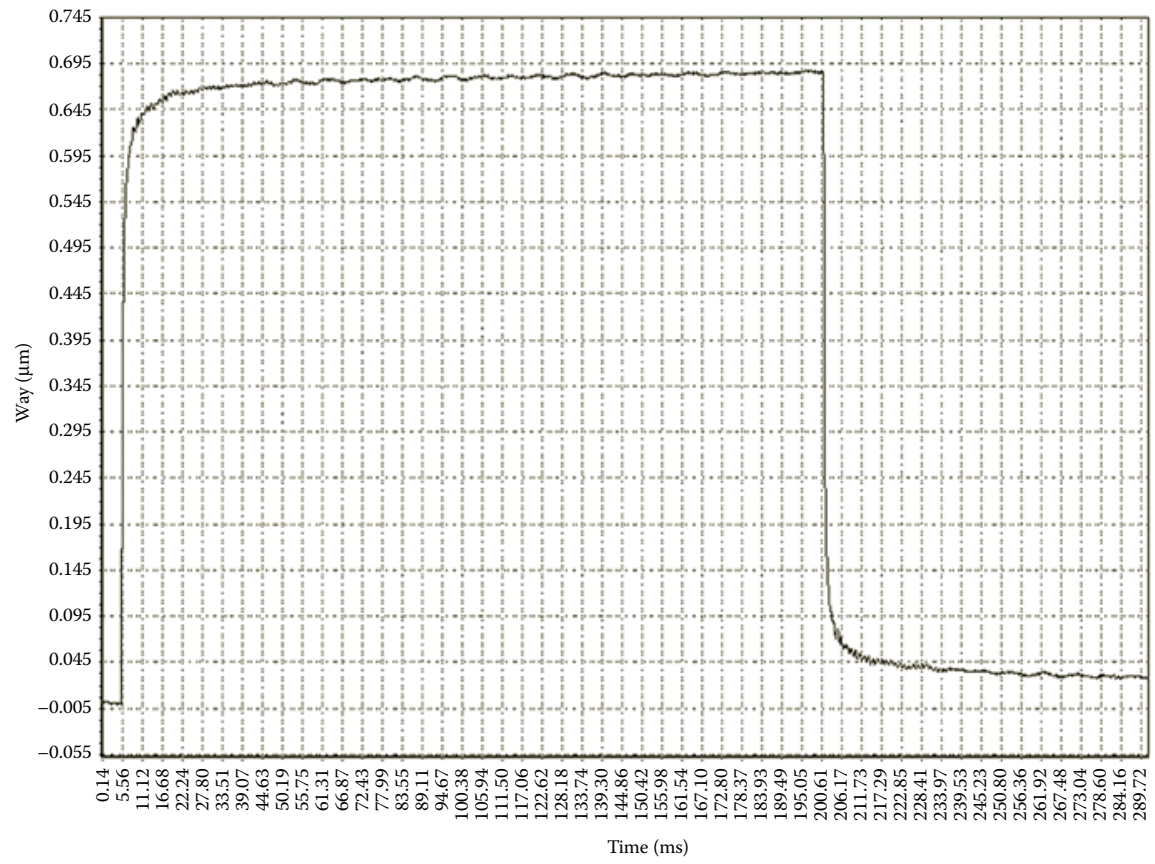

FIGURE 13.36

Closed loop system after tuning for additional load and minimum noise. 
application (i.e., fine tuning the grating position for structured illumination) might require the lowest possible noise with no speed requirement. In most cases manufacturers can tune the system for a particular loading configuration, motion waveform, and desired resolution or speed.

Both types of sensors-strain gage and capacitive-have properties which will be unique to the stage system for which they are being used. Every strain gage and capacitive sensor system will have different gain and offset parameters even for identical stage systems. For strain gages these parameters are determined by resistance tolerances and prestressing of the metal flexure. Capacitive sensors are affected by offset distance and angular alignment.

For OEM systems this is often problematic since parts of a system (i.e., stage, cables, electronics) cannot be interchanged and any failure in one part of the system would necessitate complete replacement of all parts or at the very least, a recalibration for gain and offset parameters by an interferometer. Therefore modern piezoelectric closed loop systems offer integrated precalibrated stage systems with some calibration data stored in the stage or stage-cable assembly. This may be as simple as using potentiometers to regulate the voltage outputs to more complete data-chip storage systems which include PID parameters for the closed loop system. The two architectures discussed are more easily understood with the following diagrams:

\section{Config. 1.}

At motion site

At control site

Stage -------------------- voltage cable Amplifiers+

$+$

PID+ Calibration Data

Sensor sensor signal cable conditioning electronics

\section{Config. 2.}

At motion site

At control site

Stage

$+$

Sensor

$+$

Conditioning Electronics Amplifiers + PID

$+$

Calibration Data for PID

Config. 2 offers the advantage of the ability to replace the stage system or electronics without having to have the complete system for recalibration. 


\subsection{CONCLUSION}

Piezoelectric flexure systems have unique properties that require special consideration when implementing them into a design. Specifically special attention should be given to hysteresis, drift, temperature influences, damping, and electrical drive considerations. They also offer numerous advantages over traditional motorized linear drives. These include:

\section{High stiffness}

High structural resonant frequencies

Sub-nm resolution

High speed

High load capacities

Large force generation

These advantages have made them indispensible in applications that require ultraprecise positioning accuracies. Some examples follow:

\section{Configuration}

Stack type and ring actuators

Single-axis stages with mounting platforms

Open frame $x-y$ stages with large central apertures

Compact multidimensional translation stages

High speed scanning stages

Mirror tilting systems

Piezo actuated slit apertures

Piezo actuated grippers

\section{Application}

Valve control, laser cavity tuning

Rapid confocal microscopy Z-stack acquisition, high resolution focusing devices, grating positioning for structured illumination microscopy

Optical microscopy, atomic force microscopy, scanning electron microscopy

Laser trapping and cooling techniques, optical tweezers, fiber optic alignment, storage device head alignment, CCD chip resolution enhancement, MEMS and microfluidic alignment tools

Rapid prototyping machines

Laser alignment, interferometric systems

Scanning electron microscope, proton beam, and laser aperture control, spectroscopy

Cleanroom pick and place devices

As the need for smaller and smaller system continues at a rapid pace, the need for piezoelectric positioning solutions continues to grow.

\section{REFERENCES}

1. Schmidt, R.; Waller, H. Schwingungslehre für Ingenieure-Theorie, Simulation, Anwendungen; Wissenschaftsverlag Mannheim/Wien/Zürich, 1989.

2. Götz, B.; Martin, T.; Duparre, J.W.; Bücker, P. Theoretische und experimentelle Untersuchung relevanter Parameter von Piezoaktoren; Technischer Report, Piezosystem Jena, 1998.

3. Wittenburg, J. Schwingungslehre; Springer Verlag 1996. 
4. Borchhardt, G.; Wehrsdorfer, E.; Karthe, W.; Hertsch, P.; Höfer, B. Displacement amplification mechanism for dynamic use; Technical report, Fraunhofer Institute of Applied Optics and Precision Engineering, 1998.

5. Müller, R. Verbesserung des Einschwingverhaltens wegübersetzter piezoelektrischer Aktoren durch Optimierungder mechanischen, dynamischen Parameter; degree dissertation FH-JenaUniversity of Applied Sciences, Piezosystem Jena, Jena, 2005.

6. Lorenz, M. Optimierung des Einschwingverhaltens piezogetriebener Einachsenmikropositioniertische mittels Integration eines passiven Dämpfungsgliedes; degree dissertation FH-JenaUniversity of Applied Sciences, Piezosystem Jena, Jena, 2006. 